\title{
Influence of electron-phonon interaction and crystal field on thermal and electrical resistivity in rare earth intermetallics
}

\author{
Krzysztof Durczewski, Zbigniew Gajek ${ }^{\mathrm{a}}$, and Jan Mucha \\ W. Trzebiatowski Institute of Low temperature and Structure Research, Polish Academy of Sciences, \\ P.O. Box 937, 50-950 Wroclaw, Poland
}

Received 1 February 2020 / Received in final form 29 March 2020

Published online 25 May 2020

(C) The Author(s) 2020. This article is published with open access at Springerlink.com

\begin{abstract}
The thermal and electrical resistivity of f-electron cubic intermetallic metals with well localized and stable stable f-shells are calculated as functions of the temperature. The interaction of the conduction electron with both acoustic and optical phonons as well as the exchange $\mathrm{s}-\mathrm{f}$ interaction with the crystal field presence are taken into account by applying the Matthiessen rule on the microscopic level and simple well-founded models of the scattering with the same mathematical method within the Boltzmann equation approach. Derived analytical formulas are successfully used to model existing measurements of temperature dependence of electric and thermal conductivity for $\mathrm{DyIn}_{3}$ and $\mathrm{TmIn}_{3}$ in the paramagnetic phase. Attention was drawn to the possible indeterminacy of some material constants in the absence of indications from sources other than transport measurements, noticeable in our results for $\operatorname{TmIn}_{3}$.
\end{abstract}

\section{Introduction}

Though the physics of rare earth compounds is recently focused on ones of unstable 4f-shells, however, certain problems of the physics of the compounds of stable $4 \mathrm{f}-$ shells have been left aside. Such a problem is the behavior of the thermal conductivity or thermal resistivity of the rare earth intermetallics of stable and localized 4f-shells, which we shall call "normal". The closely related topic of their electrical resistivity has been worked out rather thoroughly.

The exchange s-f interaction [1] and the crystal field splitting (see, e.g. [2,3]) of the ground state f-electron multiplet plays an important role in the conduction electron scattering responsible for the magnitude of the transport coefficients in rare earth intermetallics at low and moderate temperatures up to 100-200 K depending on the total crystal field splitting. In order to compare the magnitude of the theoretical values of these coefficients to experimental data in a wider temperature range up to the room temperature one has also to consider the scattering of the conduction electrons by phonons and impurities.

In [4] on the basis of a crystal-field model adequate for cubic compounds and the standard treating of the interaction of the conduction electrons with acoustic phonons and with impurities the thermal resistivity and electrical resistivity as functions of the temperature were calculated and fitted to experimental data of $\mathrm{DyIn}_{3}$. Very approximate treatment of the scattering by optical phonons was at issue of that study. In the present paper we calculate functions describing contribution of the interaction

\footnotetext{
${ }^{\mathrm{a}}$ e-mail: z.gajek@intibs.pl
}

of the conduction electrons with the optical phonons both to the electrical and thermal conductivity in metals. The formulas are developed on the same footing as the remaining contributions considered earlier, derivation of which is also sketched shortly for completeness. Similarly as in our previous short paper [4] we restrict the considerations to the electronic conduction while treating theoretically the thermal and electrical resistivity. The dominating role of the electron transport of heat over the phonon conductivity is clearly seen in recent ab initio approaches based on density functional theory for a wide group of noble metals and intermetallic compounds, although not negligible in all cases (see for instance [5] and references therein). Comparison of the heat and electric transport in $\mathrm{REIn}_{3}$ indicates that the contribution of other than the electronic carriers does not exceed a few percent [6].

There are very rare papers in which the scattering of the conduction electrons by optical phonons in metals has been taken into account to describe its influence to the transport coefficients in metals. This influence is not mentioned in the review articles such as [7]. It is rather considered that optical phonons are important in the transport in semiconductors (see, e.g. [8]). According our knowledge there are only few papers in which the optical phonon scattering contribution was taken into account to the electrical resistivity of certain materials [9-11]. In the first two papers the authors used the formula derived for semiconductors [12] in the limiting case of the degenerate electron gas.

Electrical and thermal currents in normal f-electron metals can be considered to be carried by s-type electrons. As concerns the scattering mechanisms in them 
there does not exist a single dominant one allowing to neglect the others, as it is in the case of some "anomalous" f-electron intermetallics in which the f-shell is "unstable" thereby having f-electrons participating in the conduction process. To take into account several scattering sources in [4], we applied the Matthiessen rule on the microscopic level and expressed the electrical and thermal resistivity as linear combinations of dimensionless functions describing the contributions of these sources. Thus, for both transport coefficients we write down this combination in the following way

$$
\begin{aligned}
\rho(T)= & \rho_{\text {imp }}+\rho_{\mathrm{o}}^{(\mathrm{ac})} \mathcal{F}_{11}\left(t_{\mathrm{D}}\right)+\rho_{\mathrm{o}}^{(\mathrm{op})} \mathcal{G}_{11}\left(t_{\mathrm{E}}\right)+\rho_{\mathrm{o}}^{(\mathrm{sf})} \mathcal{R}_{\mathrm{J}}(T) \\
W(T)= & \mathcal{W}_{\text {imp }}(T)+\left(\rho_{\mathrm{o}}^{(\mathrm{ac})} / \widetilde{\mathcal{L}}_{\mathrm{o}} T\right) \mathcal{F}_{22}\left(t_{\mathrm{D}}\right) \\
& +\left(\rho_{\mathrm{o}}^{(\mathrm{op})} / \widetilde{\mathcal{L}}_{\mathrm{o}} T\right) \mathcal{G}_{22}\left(t_{\mathrm{E}}\right)+\left(\rho_{\mathrm{o}}^{(\mathrm{sf})} / \widetilde{\mathcal{L}}_{\mathrm{o}} T\right) \mathcal{W}_{\mathrm{J}}(T), \quad(1)
\end{aligned}
$$

where the impurity scattering contribution $\rho_{\text {imp }}$ to the electrical resistivity and the impurity thermal resistivity are interrelated $\mathcal{W}_{\mathrm{imp}}(T)=\rho_{\mathrm{imp}}\left(\mathcal{L}_{\mathrm{o}} T\right)^{-1} \cdot \mathcal{L}_{\mathrm{o}}=\left(\pi^{2} / 3\right)$. $\left(k_{\mathrm{B}} / e\right)^{2} \approx 2.4462 \cdot 10^{-8} \Omega \mathrm{W} / \mathrm{K}^{2}$ is the free electron (Sommerfeld) Lorenz number. In front of the functions of $W(T)$ describing other scattering sources there is the factor $\left(\widetilde{\mathcal{L}}_{\mathrm{o}} T\right)^{-1}$, where $\widetilde{\mathcal{L}}_{\mathrm{o}}=\left(\pi^{2} / 3\right) \mathcal{L}_{\mathrm{o}}$.

The magnitude of $\rho_{\text {imp }}$ is considered as a phenomenological constant. We do not consider the impurity scattering in a microscopical way, e.g., such as in [13]. The functions $\mathcal{F}_{11}\left(t_{\mathrm{D}}\right), \mathcal{F}_{22}\left(t_{\mathrm{D}}\right)$ of the argument $t_{\mathrm{D}}=T / T_{\mathrm{D}}$ with the Debye temperature $T_{\mathrm{D}}$ describe the scattering of the conduction electrons by acoustic phonons. Similarly, the functions $\mathcal{G}_{11}\left(t_{\mathrm{E}}\right)$ and $\mathcal{G}_{22}\left(t_{\mathrm{E}}\right)$ dependent on $t_{\mathrm{E}}=T / T_{\mathrm{E}}$ with the Einstein temperature $T_{\mathrm{E}}$ express the scattering by optical phonons. The spin dependent s-f scattering is described by the functions $\mathcal{R}_{\mathrm{J}}(T), \mathcal{W}_{\mathrm{J}}(T)$; the index $J$ being a parameter of these functions stands for the magnitude of the total angular momentum $J$ of $4 \mathrm{f}$-shell.

In Section 2 we shortly describe the mathematical method applied to deriving the functions of (1), then in the introductory part of Section 3 we show how this method is applied to the scattering of the conduction electrons by phonons. The model assumptions and the basic approximations which lead to the final form of the functions $\mathcal{F}_{11}\left(t_{\mathrm{D}}\right)$ and $\mathcal{F}_{22}\left(t_{\mathrm{D}}\right)$, i.e.,in the case of the acoustic phonons, are presented in Section 3.1. The same for the case of the functions $\mathcal{G}_{11}\left(t_{\mathrm{E}}\right)$ and $\mathcal{G}_{22}\left(t_{\mathrm{E}}\right)$, i.e., the optical phonons, is described in Section 3.2. The derivations of formulas for $\mathcal{R}_{\mathrm{J}}(T)$ and $\mathcal{W}_{\mathrm{J}}(T)$ are found in Section 4. We restrict our considerations to paramagnetic phase and only indicate how the magnetic order enters the final expressions for $\mathcal{R}_{\mathrm{J}}(T)$ and $\mathcal{W}_{\mathrm{J}}(T)$ in ferromagnets. The mentioned restriction in the case of REIn ${ }_{3}$ crystals still means a wide temperature interval. The thermal conductivity and electrical resistivity of $\mathrm{REIn}_{3}$ monocrystals were experimentally examined in [6] up to about $300 \mathrm{~K}$; their transition temperatures (mainly to antiferromagnetic phases) are rather low and do not exceed $20 \mathrm{~K}$. To avoid complications caused by effects of a magnetic order on the crystal field we take into account the crystalline electric field only in the paramagnetic phase. The asymptotic values of $\mathcal{R}_{\mathrm{J}}(T)$ and $\mathcal{W}_{\mathrm{J}}(T)$ of our model
RE-monocrystal are discussed in a separate section (Sect. 5). For an illustration how our results agree with experimental ones we show in Section 6 the fitting of the total theoretical electrical and thermal resistivities to the experimental data of $\operatorname{DyIn}_{3}$ and $\operatorname{TmIn}_{3}$ of the Néel temperatures $T_{\mathrm{N}}=20 \mathrm{~K}$ and $T_{\mathrm{N}}=1.6 \mathrm{~K}$, respectively, according recent data (see [14] and references therein).

According to our knowledge the method in which the s-f contributions to the electrical and thermal resistivity is calculated on the same footing as in the present paper and [4] has never be applied before. The s-f contribution to the electrical resistivity has been calculated traditionally by using the crystal-field level scheme and corresponding wave functions known from inelastic neutron scattering experiments data. The results have usually been compared to the experimental s-f contribution found as the difference between the measured total electrical resistivity of the examined RE monocrystal and the phonon contribution considered as the measured electrical resistivity of an isomorphous nonmagnetic compound (see, e.g. [15]). In the case of $\mathrm{REIn}_{3}$ the phonon scattering resistivity standard is the electrical resistivity of $\mathrm{LaIn}_{3}$ or $\mathrm{LuIn}_{3}$. As concerns the thermal resistivity there are only a few papers $[6,16,17]$ in which the contribution "due to spin dependent scattering" was extracted from the total thermal resistivity.

It is also worth paying reader's attention that Section 3.2 contains a reconsideration of the paper by Howarth and Sondheimer [12] in which the simple Fröhlich model [18] was applied to scattering of the conduction electrons in polar semiconductors. The variational principle used in [12] to solve the Boltzmann equation is also applied in our paper but in a simpler version, appropriate to metals. Certain, less essential, formulas of this section, of mathematical but not physical significance, are shifted to Appendix B.

Appendix A contains a remark on the correction of the formula describing the scattering by acoustic phonons in [4]. In Appendix C one can find the expressions for the material constants of (1) which follow from all our models of the scattering.

\section{The Ziman variational method of solving the transport problem}

The linear combinations of the functions in (1) are a consequence of the fact that the transition probabilities describing the corresponding scattering problems are considered in the first approximation to be additive. This assumption is the Matthiessen rule for the electrical and thermal conductivity on the microscopic level. It can be justified by an appropriate perturbation technique [19]. The generalized Matthiessen rule was also used in an already "classical" paper on the magnetic neutron scattering [20] and in the monographs [21,22]. The additive contribution of the scattering arising from the acoustic and optical phonons (1) is simply a consequence of the fact that the electron-phonon interaction potential corresponding to different phonon branches is additive.

Under the assumption of the generalized Matthiessen rule one can consider separately each scattering 
problem relevant to finding a function in (1). Let $C\left(\mathbf{k}, \mathbf{k}^{\prime} ; T\right)$ denotes the transition probability of a scattering with the transition of the conduction electron of the wave vector $\mathbf{k}$ to the state of the vector $\mathbf{k}^{\prime}$. The matrix

$$
\begin{aligned}
P_{i j}= & \frac{1}{2} \int \mathrm{d} \mathfrak{v}_{\mathbf{k}} \mathrm{d} \mathfrak{v}_{\mathbf{k}}^{\prime} C\left(\mathbf{k}, \mathbf{k}^{\prime} ; T\right)\left[\chi_{i}(\mathbf{k})-\chi_{i}\left(\mathbf{k}^{\prime}\right)\right] \\
& \times\left[\chi_{j}(\mathbf{k})-\chi_{j}\left(\mathbf{k}^{\prime}\right)\right]
\end{aligned}
$$

proposed by Ziman [23], who took advantage of the Kohler variational formalism [24-26] of an approximate of the Boltzmann equation, allows one to derive the relevant function of (1). The integral is with respect to $d \mathfrak{v}_{\mathrm{k}}=$ $\mathrm{d} k_{x} \mathrm{~d} k_{y} \mathrm{~d} k_{z}$. The functions $\chi_{i}(\mathbf{k})=\left(\mathbf{v}_{\mathbf{k}} \mathbf{u}\right)(\epsilon-\zeta)^{i-1}(i=$ $1,2)$ are components of microscopic electric and thermal currents in the direction (unit vector $\mathbf{u}$ ) of the external electric field or the thermal gradient. ${ }^{1}$ The chemical potential of the conduction electrons, $\zeta=\zeta(T)$, is counted from the bottom of the conduction band. The transition probability $C\left(\mathbf{k}, \mathbf{k}^{\prime} ; T\right)$ has the following structure

$$
C\left(\mathbf{k}, \mathbf{k}^{\prime} ; T\right)=\left(k_{\mathrm{B}} T\right)^{-1} f_{0}(\epsilon)\left[1-f_{0}\left(\epsilon^{\prime}\right)\right] \mathfrak{C}(\mathbf{k}, \mathbf{q}),
$$

where $\mathbf{q}=\mathbf{k}^{\prime}-\mathbf{k}$ and $\mathrm{d} \mathfrak{v}_{\mathbf{q}}=\mathrm{d} q_{x} \mathrm{~d} q_{y} \mathrm{~d}_{z}, \epsilon=\epsilon(\mathbf{k}), \epsilon^{\prime}=$ $\epsilon\left(\mathbf{k}^{\prime}\right), f_{0}(\epsilon)\left[1-f_{0}\left(\epsilon^{\prime}\right)\right]$ and $\mathfrak{C}(\mathbf{k}, \mathbf{q}) . \mathrm{C}\left(\mathbf{k}, \mathbf{k}^{\prime} ; \mathrm{T}\right)$ has to be calculated in the first Born approximation for an assumed model described by the relevant scattering potential.

Being interested in metals we confine our considerations to the degenerate electron gas. In this case the electrical resistivity is expressed by $P_{11}[23,28]$ and the thermal resistivity by $P_{22}[13,23]$ as it is presented in (1). In general case the electrical and thermal conductivity are expressed by all the components of the scattering matrix $P_{i j}$ being functions of $z=\zeta / k_{\mathrm{B}} T$ and $T$ explicitly and depending as well on the macroscopic currents $\left.J_{i}(z), U_{i}(z)[23,28]\right)$. In the "metallic limit", $z \rightarrow \infty$, the currents can be represented by in terms of the material constant $U_{0}$ (see Sect. C.2 in Appendix C) or $J_{0}=e U_{0}$ and $\zeta \approx \epsilon_{\mathrm{F}}$, where $\epsilon_{\mathrm{F}}$ is the Fermi energy. The matrix elements $P_{i j}$ in this approximation can be represented

$$
P_{i j}(T)=\lim _{z \rightarrow \infty} P_{i j}(z, T)=\mathcal{P}_{0}\left(k_{\mathrm{B}} T\right)^{i+j-2} \mathcal{P}_{i j}(T),
$$

where $\mathcal{P}_{0}$ is a material constant for the considered scattering source and $\mathcal{P}_{i j}(T)$ is a dimensionless function. The values of $\mathcal{P}_{0}$ for the considered scattering types are presented in Appendix C. The functions $\mathcal{P}_{i j}(T)$ are denoted $\mathcal{F}_{i j}\left(t_{\mathrm{D}}\right)$ and $\mathcal{G}_{i j}\left(t_{\mathrm{E}}\right)$ for the scattering by acoustic and optical phonons, respectively. In case of the s-f scattering only diagonal components $\mathcal{P}_{11}(T)=\mathcal{R}_{\mathrm{J}}(T)$ and $\mathcal{P}_{22}(T)=$ $\mathcal{W}_{\mathrm{J}}(T)$ are essential for our considerations.

In order to derive $P_{i j}(z, T)$ one has to represent the integrands of (2) as functions of $\epsilon$ and to calculate the integrals (2) with respect $\epsilon$ in the limits $(0, \infty)$ for sufficiently large values $\zeta \approx \epsilon_{\mathrm{F}}$ with respect the bottom of the

\footnotetext{
${ }^{1}$ We define the trial functions here in a more general way than in $[27,28]$, namely $\chi_{i}(\mathbf{k})=\left(\mathbf{v}_{\mathbf{k}} \mathbf{u}\right)(\epsilon-\zeta)^{i-1}$ instead of $(\mathbf{k} \mathbf{u})(\epsilon-\zeta)^{\mathbf{i}-\mathbf{1}}$. For $\epsilon(\mathbf{k})=\left(\hbar^{2} / 2 m\right) \mathbf{k}^{2}$ this cause changes only in additional factors in $\rho_{\mathrm{O}}^{(\mathrm{ac})}, \rho_{\mathrm{O}}^{(\mathrm{op})}, \rho_{\mathrm{O}}^{(\mathrm{sf})}$ of $(1)$, but not in the final values of $\rho(T)$ and $W(T)$, see Appendix B in [13].
}

conduction band. The basic contribution to these integrals comes from the nearest vicinity of $\zeta$. It is seen, e.g. from the identity

$$
\left(k_{\mathrm{B}} T\right)^{-1} f_{0}(\epsilon)\left[1-f_{0}\left(\epsilon^{\prime}\right)\right] \equiv\left(-\frac{\partial f_{0}}{\partial \epsilon}\right) \mathfrak{G}\left(\epsilon, \epsilon^{\prime}\right),
$$

where

$$
\mathfrak{G}\left(\epsilon, \epsilon^{\prime}\right)=\frac{1}{1-f_{0}(\epsilon)\left[1-\exp \left(\epsilon-\epsilon^{\prime}\right) / k_{\mathrm{B}} T\right]},
$$

since $-\partial f_{0} / \partial \epsilon \approx \delta(\epsilon-\zeta)$.

The above identity with further application of the Sommerfeld expansion in the powers of $(\epsilon-\zeta)^{n}$ in the intergrand of integrals with respect the electron energy up to the lowest order terms (see, e.g. [23,29]) was applied by us in [27]. A better approximation is usually applied when the scattering of the conduction electrons by phonons is considered. It was proposed by Wilson [26] and we used it in [28] and shortly describe in the next section.

The formulas (1) follow from applying the generalized Matthiessen rule to the scattering by the sources described in Sections 3 and 4. The relevant components of the sums (1) are derived from the general expressions for the transport coefficients of the Ziman method [13,23,28] after taking the limit of (4) and representing the currents $J_{i}(z)$, $U_{i}(z)[13,28]$ as series in powers of $z^{-1}$ and appropriate abbreviation. In this way one obtains

$$
\rho(T)=\frac{\mathcal{P}_{0}}{e^{2} U_{0}^{2} \epsilon_{\mathrm{F}}^{3}} \mathcal{P}_{11}(T) \frac{1-\mathcal{P}_{12}^{2} /\left(\mathcal{P}_{11} \mathcal{P}_{22}\right)}{1+O\left(z^{-1}\right)} \approx \rho_{\mathrm{o}} \mathcal{P}_{11}(T),
$$

where $O\left(z^{-n}\right)$ means a quantity at most of the order $z^{-n}$ and $U_{0}^{2}$ is a constant simply expressed by the effective electron mass and the Planck constant (see Appendix B in [13]). In the final formula $\mathcal{P}_{12}^{2} / \mathcal{P}_{11} \mathcal{P}_{22}$ is neglected since $\mathcal{P}_{12}^{2} \ll \mathcal{P}_{11} \mathcal{P}_{22}$, as it will be seen from our final considerations in Sections 3 and 4. The formula of (1) for the thermal resistivity is a consequence of the considerations of [13], where $W$ was represented as

$$
W(T)=\frac{\mathcal{P}_{\mathrm{o}}}{U_{0}^{2} \epsilon_{\mathrm{F}}^{3}} \frac{T \mathcal{P}_{22}(T)}{\left(k_{\mathrm{B}} T\right)^{2}\left[\left(\pi^{2} / 3\right)^{2}+O\left(z^{-2}\right)\right]} \approx \frac{\rho_{\mathrm{o}}}{T \widetilde{\mathcal{L}}_{\mathrm{o}}} \mathcal{P}_{22}(T) .
$$

The validity of the (generalized) Matthiessen rule is of principal importance to the validity of the above equations. The cases of the violation of the Matthiessen rule are discussed in Section 5 of [13].

\section{The scattering of conduction electrons by phonons}

It is known that the function $\mathfrak{C}(\mathbf{k}, \mathbf{q})$ in (3) can be represented in the following way

$$
\begin{aligned}
\mathfrak{C}(\mathbf{k}, \mathbf{q})= & \mathfrak{C}_{0}\left(\omega_{\mathbf{q}}, \mathbf{q}\right)\left[\mathcal{N}_{\mathbf{q}} \delta\left(\epsilon^{\prime}-\epsilon-\hbar \omega_{\mathbf{q}}\right)\right. \\
& \left.+\left(\mathcal{N}_{\mathbf{q}}+1\right) \delta\left(\epsilon^{\prime}-\epsilon+\hbar \omega_{\mathbf{q}}\right)\right] \\
\equiv & \mathfrak{C}_{\mathrm{a}}(\mathbf{k}, \mathbf{q})+\mathfrak{C}_{\mathrm{e}}(\mathbf{k}, \mathbf{q}),
\end{aligned}
$$


when the conduction electrons are scattered by phonons of the spectrum $\omega_{\mathrm{a}}$. In [30] the Boltzmann equation and the above expression were derived from basic principles of the statistical mechanics. Besides the energy conservation laws expressed by the arguments of the $\delta$ functions, the phonon spectrum enters the distribution function $\mathcal{N}_{\mathbf{q}}=$ $\left[\exp \left(\hbar \omega_{\mathbf{q}} / k_{\mathrm{B}} T\right)-1\right]^{-1}$. The first component of $\mathfrak{C}(\mathbf{k}, \mathbf{q})$ describes the process of the phonon absorption, the second expresses its emission. The electron momentum conservation law is $\mathbf{q}=\mathbf{k}^{\prime}-\mathbf{k}$ since we neglect the Umklapp process in our further considerations.

The application of the formula (9) is usually limited to the scattering by acoustic phonons. In [12], this form of $\mathfrak{C}(\mathbf{k}, \mathbf{q})$ was also applied to study the influence of the scattering of the conduction electrons by optical phonons on the electrical resistivity in polar semiconductors.

In the present paper - by assuming the following phonon spectra

$$
\hbar \omega_{\mathbf{q}}= \begin{cases}\hbar v_{\mathrm{s}} q & \text { of acoustic phonons, Section 3.1 } \\ \hbar \omega_{0} \equiv k_{\mathrm{B}} T_{\mathrm{E}} & \text { of optical phonons, Section 3.2 }\end{cases}
$$

with $v_{\mathrm{s}}$ the sound velocity, $q=|\mathbf{q}|$ and $T_{\mathrm{E}}$ is the Einstein temperature - we apply the transition probability (9) for studying the scattering the conduction electrons by phonons.

Before calculating the integrals $P_{i j},(2)$, it is convenient to neglect in advance all the terms linear in $\mathbf{k}$ and $\mathbf{k}^{\prime}$ in the integrands, which do not contribute to the integrals. In this way one can consider as a starting formulas of calculating the integrals $P_{11}, P_{12}=P_{21}, P_{22}$ the following integrals

$$
\begin{aligned}
P_{11}= & \frac{1}{2} \int \mathrm{d} \mathfrak{v}_{\mathbf{k}} \mathrm{d} \mathfrak{v}_{\mathbf{q}} C(\mathbf{k}, \mathbf{k}+\mathbf{q} ; T)(\mathbf{q} \mathbf{u})^{2}, \\
P_{12}= & \frac{1}{2} \int \mathrm{d} \mathfrak{v}_{\mathbf{k}} \mathrm{d} \mathfrak{v}_{\mathbf{q}} C(\mathbf{k}, \mathbf{q} ; T)\left[(\epsilon-\zeta)+\left(\epsilon^{\prime}-\epsilon\right)\right](\mathbf{q} \mathbf{u})^{2}, \\
P_{22}= & \frac{1}{2} \int \mathrm{d} \mathfrak{v}_{\mathbf{k}} \mathrm{d} \mathfrak{v}_{\mathbf{q}} C(\mathbf{k}, \mathbf{k}+\mathbf{q} ; T)\left\{( \mathbf { q } \mathbf { u } ) ^ { 2 } \left[(\epsilon-\zeta)^{2}\right.\right. \\
& \left.\left.+2\left(\epsilon^{\prime}-\epsilon\right)(\epsilon-\zeta)+\left(\epsilon^{\prime}-\epsilon\right)^{2}\right]+\left(\epsilon^{\prime}-\epsilon\right)^{2}(\mathbf{k} \mathbf{u})^{2}\right\} .
\end{aligned}
$$

After performing the above integrals with respect the angles in the spherical coordinates (for instance in the way described in [28]), we obtain

$$
\begin{aligned}
P_{i j} & =P_{\mathrm{o}}\left(1 / k_{\mathrm{B}} T\right) \\
& \times \int_{0}^{\infty} \mathrm{d} \epsilon \int_{\mathfrak{a}}^{\mathfrak{b}} \mathrm{d} q f_{0}(\epsilon)\left[1-f_{0}\left(\epsilon+\hbar \omega_{\mathbf{q}}\right)\right] \mathcal{N}_{\mathbf{q}} \mathcal{V}_{\mathrm{ij}}^{(\mathrm{a})}(\epsilon, q) \\
& +P_{\mathrm{o}}\left(1 / k_{\mathrm{B}} T\right) \\
& \times \int_{0}^{\infty} \mathrm{d} \epsilon \int_{\mathfrak{c}}^{\mathfrak{d}} \mathrm{d} q f_{0}(\epsilon)\left[1-f_{0}\left(\epsilon-\hbar \omega_{\mathbf{q}}\right)\right]\left(\mathcal{N}_{\mathbf{q}}+1\right) \mathcal{V}_{\mathrm{ij}}^{(\mathrm{e})}(\epsilon, q) \\
& \equiv P_{\mathrm{o}}\left(P_{i j}^{(\mathrm{a})}+P_{i j}^{(\mathrm{e})}\right)
\end{aligned}
$$

where $P_{\mathrm{o}}$ is a material constant. The lower $\mathfrak{a}, \mathfrak{c}$ and upper $\mathfrak{b}, \mathfrak{d}$ integral limits are respectively the minimal and maximal values of $q=|\mathbf{q}|$ allowable by the energy conservation laws. They can depend in general on the conduction electron energy. The functions $\mathcal{V}_{\mathrm{ij}}^{(\mathrm{a})}, \mathcal{V}_{\mathrm{ij}}^{(\mathrm{e})}$ in the integrands are

$$
\begin{aligned}
\mathcal{V}_{11}^{(\mathrm{a})}(\epsilon, q)= & \mathcal{V}_{11}^{(\mathrm{e})}(\epsilon, q)=q^{l+3}, \\
\mathcal{V}_{12}^{(\mathrm{a}, \mathrm{e})}(\epsilon, q)= & {\left[(\epsilon-\zeta)+\eta \hbar \omega_{\mathbf{q}}\right] q^{l+3}, } \\
\mathcal{V}_{22}^{(\mathrm{a}, \mathrm{e})}(\epsilon, q)= & {\left[(\epsilon-\zeta)^{2}+2 \eta \hbar \omega_{\mathbf{q}}(\epsilon-\zeta)+\left(\hbar \omega_{\mathbf{q}}\right)^{2}\right] q^{l+3} } \\
& +\left(\hbar \omega_{\mathbf{q}}\right)^{2} k^{2} q^{l+1},
\end{aligned}
$$

where the index $(a)$ stands for the phonon absorption and $(e)$ for the emission and $\eta= \pm 1$. The double index $(a, e)$ means $(a)$ if $\eta=1$ and $(e)$ if $\eta=-1$. The exponents $l+1$, $l+3$ are different in the case of the scattering by acoustic or by optical phonons. The magnitude of $l$ simply follows from the dependence $\mathfrak{C}(\mathbf{k}, \mathbf{q})$ on $q$ which is presented in the following subsections.

In order to calculate the integrals with respect to $\epsilon$ and to simplify the integrals with respect to $q$, we introduce the dimensionless variables $y, p$ and the parameter $z$

$$
y=(\epsilon-\zeta) / k_{\mathrm{B}} T, \quad p=\hbar \omega_{\mathrm{q}} / k_{\mathrm{B}} T, \quad z=\zeta / k_{\mathrm{B}} T
$$

enabling to express the identity [26] as

$$
f_{0}(\epsilon)\left[1-f_{0}\left(\epsilon+\eta \hbar \omega_{\mathbf{q}}\right)\right] \equiv \frac{1}{1-\mathrm{e}^{-\eta p}} \mathfrak{F}(y, \eta p),
$$

where $\eta=\mp 1$ and

$$
\mathfrak{F}(y, \eta p)=\frac{1}{1+\mathrm{e}^{-y-\eta p}}-\frac{1}{1+\mathrm{e}^{-y}} .
$$

The above representation allows one to find the limit (4) without applying the Sommerfeld expansion unlike in the case of the representation (5).

\subsection{Acoustic phonons}

In this subsection, we consider the scattering of the conduction electrons by the acoustic phonons. Thus, we assume the function $\mathfrak{C}\left(\omega_{\mathbf{q}}, \mathbf{q}\right)$, $(9)$, as in $[27,28]$ in the simplest quasi-phenomenological approximation of the deformation potential. Within this approximation only the longitudinal phonon waves contribute to the scattering (see, e.g., Eq. (3.3) in [27]). Since the crystal is treated as a continuous medium, the transition probability is assigned to the volume of the unit cell of the crystal. For $\mathfrak{C}_{0}\left(\omega_{\mathbf{q}}, \mathbf{q}\right)$ we obtain

$$
\mathfrak{C}_{0}\left(\omega_{\mathbf{q}}, \mathbf{q}\right)=\frac{V_{\mathrm{c}}}{4 \pi^{2}} \frac{E_{1}^{2} q^{2}}{M \omega_{\mathbf{q}}},
$$

where $M$ is the mass of the unit cell of the volume $V_{\mathrm{c}}=V / N_{\mathrm{c}}, V$ and $N_{\mathrm{c}}$ is the volume of the crystal and the number of the unit cells, respectively. $E_{1}$ is a constant of the dimension of the energy, the magnitude of 
which is a few $\mathrm{eV}$. The factor $2 / 8 \pi^{2}$ in the above expression comes from taking into account two electron bands of opposite spins and considering integrals instead of sums in the Boltzmann equation and consequently in (2). Similar expression to $\mathfrak{C}_{0}\left(\omega_{\mathbf{q}}, \mathbf{q}\right) \sim \epsilon_{\mathrm{F}}^{2} q$ is obtained in case of wholly microscopic approach to the electron-phonon interaction (see e.g. [7]). Thus, the proper index $l$ in (13) is $l=1$.

The limits of integration with respect to $q$ in (12) following from the energy conservation are: the lower ones $\mathfrak{a}=\mathfrak{c}=0$, whereas the upper limits $\mathfrak{c}$ and $\mathfrak{d}$ are the electron energy dependent since the largest allowable angle between $\mathbf{k}^{\prime}$ and $\mathbf{k}$ is the electron energy dependent (see Eq. (3.90) in [28]) or equation (12a-12b) in [12]. From a physical point of view, such limits should be taken into account when the Fermi sphere is included within the boundaries of the Brillouin zone. However, since we assume the free electron approximation, the value of $q=\left|\mathbf{k}^{\prime}-\mathbf{k}\right|$ is not limited by the Brillouin boundaries. Thus, if the Fermi surface crosses these boundaries which the case occurs in metals - we have to introduce physical constraints. The simplest is the assumption that $q \leq 2 q_{\mathrm{D}}$, where $q_{\mathrm{D}}$ is the radius of the Debye sphere i.e. the sphere of the volume equal to that of the Brillouin zone. We introduce such values of $q_{\mathrm{D}}$ while considering optical phonons in the next subsection. For acoustic phonons the standard procedure is introducing the magnitude of $q_{\max }=2 q_{\mathrm{D}}$ as the cutoff value of the wave vector following from the relation $\hbar v_{\mathrm{s}} q_{\max }=k_{\mathrm{B}} T_{\mathrm{D}}$, where $T_{\mathrm{D}}$ is the Debye temperature.

On such physical assumptions one finds the metallic limit (4) of the matrix elements $P_{i j}(z, T)$ after taking into account the the identity (15), changing the order of the integration with respect $q$ i $\epsilon$ in (12) and using, e.g., the method of Appendix B in [28]) to finally find the limit. The resulting matrix elements $\mathcal{P}_{i j}(T),(4)$, are linear combinations of the generalized (index $n \geq 5$ ) of the Bloch-Grüneisen functions

$$
\mathcal{J}_{n}(t)=\int_{0}^{1 / t} \frac{\mathrm{d} p p^{n}}{\left(\mathrm{e}^{p}-1\right)\left(1-\mathrm{e}^{-p}\right)}=\frac{1}{4} \int_{0}^{1 / t} \frac{\mathrm{d} p p^{n}}{\sinh ^{2}(p / 2)}
$$

of the order $n=5,6,7$. The matrix elements $\mathcal{P}_{11}^{(\mathrm{a})}(T)$ and $\mathcal{P}_{11}^{(\mathrm{e})}(T)$ corresponding to the two components of $(12)$ in the metallic limit depend on $t_{\mathrm{D}}=T / T_{\mathrm{D}}$ and are

$$
\begin{aligned}
& \mathcal{P}_{11}^{(\mathrm{a})}(T)=\mathcal{P}_{11}^{(\mathrm{e})}(T)=\frac{1}{2} t_{\mathrm{D}}^{5} \mathcal{J}_{5}\left(t_{\mathrm{D}}\right) \\
& \mathcal{P}_{12}^{(\mathrm{a})}(T)=\frac{1}{2} t_{\mathrm{D}}^{5} \mathcal{J}_{6}\left(t_{\mathrm{D}}\right) \\
& \mathcal{P}_{12}^{(\mathrm{e})}(T)=-\mathcal{P}_{12}^{(\mathrm{a})}(T)
\end{aligned}
$$

The forms of the elements $\mathcal{P}_{22}^{(\mathrm{a})}(T)$ and $\mathcal{P}_{22}^{(\mathrm{e})}(T)$ are more complicated, namely

$$
\begin{aligned}
\mathcal{P}_{22}^{(\eta)}(T)= & \frac{1}{2} t_{\mathrm{D}}^{5}\left\{\frac{\pi^{2}}{3} \mathcal{J}_{5}\left(t_{\mathrm{D}}\right)+\frac{1}{3} \mathcal{J}_{7}\left(t_{\mathrm{D}}\right)\right. \\
& \left.-\eta \frac{\epsilon_{\mathrm{S}}}{2 k_{\mathrm{B}} T} \mathcal{J}_{6}\left(t_{\mathrm{D}}\right)+\frac{\epsilon_{\mathrm{F}} \epsilon_{\mathrm{s}}}{\left(k_{\mathrm{B}} T\right)^{2}} \mathcal{J}_{5}\left(t_{\mathrm{D}}\right)\right\}
\end{aligned}
$$

where $\eta=1$ for $(\eta)=($ a) and $\eta=-1$ for $(\eta)=(\mathrm{e})$. In the last term in (20), which follows from the last one in $P_{22}$ w (11), there is the product of the Fermi energy and $\epsilon_{\mathrm{s}}=2 m v_{\mathrm{s}}^{2}$. The energy $\epsilon_{\mathrm{s}} / 4$ is the minimal electron energy for the phonon emission in case of the free electron gas scattered by acoustic phonons (see [31]), namely the scattering with the emission is allowed when the electron group velocity exceeds the speed of sound.

The formulas for the the full matrix elements are obtained obviously after summing $\mathcal{P}_{i j}(T)=\mathcal{P}_{i j}^{(a)}(T)+$ $\mathcal{P}_{i j}^{(e)}(T)$. We represent them below in the form corresponding to our starting ones (1). They read

$$
\mathcal{P}_{i j}(T)=\mathcal{F}_{i j}\left(t_{\mathrm{D}}\right)
$$

where

$$
\begin{gathered}
\mathcal{F}_{11}\left(t_{\mathrm{D}}\right)=t_{\mathrm{D}}^{5} \mathcal{J}_{5}\left(t_{\mathrm{D}}\right), \quad \mathcal{F}_{12}\left(t_{\mathrm{D}}\right)=\mathcal{F}_{21}\left(t_{\mathrm{D}}\right)=0 \\
\mathcal{F}_{22}\left(t_{\mathrm{D}}\right)=t_{\mathrm{D}}^{5}\left\{\frac{\pi^{2}}{3} \mathcal{J}_{5}\left(t_{\mathrm{D}}\right)+\frac{1}{3} \mathcal{J}_{7}\left(t_{\mathrm{D}}\right)+\frac{\epsilon_{\mathrm{F}} \epsilon_{\mathrm{S}}}{\left(k_{\mathrm{B}} T\right)^{2}} \mathcal{J}_{5}\left(t_{\mathrm{D}}\right)\right\} .
\end{gathered}
$$

In Section 6 we take advantage of the above functions for an analysis of experimental data. The "model" magnitude of $\rho_{\mathrm{o}}^{\text {(ac) }}$ of (1), following from the value $\mathcal{P}_{\mathrm{o}}=\mathcal{P}_{\mathrm{o}}^{\text {(ac) }}$ of (4), is presented in Appendix C.

In our preliminary numerical analysis of $\mathrm{DyIn}_{3}$ in [4] we used the function $\mathcal{F}_{22}\left(t_{\mathrm{D}}\right)$ following from $2 \mathcal{P}_{22}^{(1)}(T)$ of (20), i.e., with the additional term $-\eta \epsilon_{\mathrm{s}} /\left(2 k_{\mathrm{B}} T\right) \mathcal{J}_{6}\left(t_{\mathrm{D}}\right)$ (see Appendix A for details). Since the energy $\epsilon_{\mathrm{S}}$ is a few $\mathrm{K}$, this term occurred to be not essential in our numerical analysis.

As concerns the electrical resistivity, the formula (22) following from (1) is the standard which requires no further comment. In the case of the thermal conductivity, there are certain differences between the formula (1), (23) and those obtained earlier by other authors for the same model but by using various approximations. Of the earlier results, one should first of all mention the Wilson formulas $[26,32]$ obtained by solving the Boltzmann equation by successive approximations. The temperature dependence of the thermal conductivity following from the formula of [32] was for the first time analyzed numerically by Makinson [33], who also took into account the scattering by static impurities in his numerical analysis and graphical representation of the results.

The formula for the thermal resistivity presented by Wilson in Chapter 9 of his monograph [26] yields

$$
\mathcal{F}_{22}\left(t_{\mathrm{D}}\right)=t_{\mathrm{D}}^{5}\left\{\frac{\pi^{2}}{3} \mathcal{J}_{5}\left(t_{\mathrm{D}}\right)+A \mathcal{J}_{7}\left(t_{\mathrm{D}}\right)+B \mathcal{J}_{5}\left(t_{\mathrm{D}}\right)\right\},
$$

where $A=-1$ and $B=\left(\epsilon_{\mathrm{F}} / 2 D\right) t_{\mathrm{D}}^{-2}$ with $D=$ $\left(6 \pi^{2}\right)^{2 / 3} \hbar^{2} /\left(4 m a^{2}\right)$ and $a$ being the lattice constant. Berman in Chapter 11 of [34] presents similar formula which implies (24) with $A=-1 / 6$ and $B=\left(k_{\mathrm{F}} / q_{\mathrm{D}}\right)^{2} t_{\mathrm{D}}^{-2}$ and refers to an earlier paper by Wilson. The function (24) is also presented in $[17,29]$. Pay attention that $D$ in the 
above Wilson expression for $\mathcal{F}_{22}\left(t_{\mathrm{D}}\right),(24)$ is an electron energy corresponding to the magnitude of the electron wave vector $q_{\mathrm{D}}$ which is estimated in the next Subsection. A similar expression was also recently obtained in [35] In this case the author obtained (24) with $A=-1 / 6$ and $B=\left(\epsilon_{\mathrm{s}} \epsilon_{\mathrm{F}} /\left(k_{\mathrm{B}} T\right)^{2}\right.$.

More sophisticated methods of the theoretical approach based on the Boltzmann equation to finding the phonon limited electrical and thermal conductivity of metals were worked out in the period of the development of numerical studies of the band structure of metals. These methods, which take into account a real band structure and an experimental phonon spectrum, are obviously purely numerical. The electron-phonon interaction is then described in terms of transport coupling function [21] which generalize the Eliashberg electron-phonon spectral function [21] used in superconductivity theory. Two methods are applied to find a numerical solution of the Boltzmann equation. The first $[21,36]$ is based on the concept of an anisotropic relaxation time $\tau(\mathbf{k}, T)[37,38]$, which in the limit to a first iteration of the integral equation for $\tau(\mathbf{k}, T)$ allow to get a simple closed expression for the scattering times at any temperature from which one can calculate the transport coefficients. The second method $[39,40]$ is based on the idea of representing the Boltzmann equation as an algebraic equation and searching for its solution by using successive approximation in terms of functions built of basis functions consisting of products of Fermi-surface harmonics and polynomials in $(\epsilon-\zeta) / k_{\mathrm{B}} T$. Both methods lead to the results from which in the first approximation one can apparently obtain formulas expressed by the Bloch-Grüneisen functions [22]. Taking into account of a few scattering sources within the purely numerical methods described above is hardly possible.

As we mentioned in the Introduction, modern purely numerical methods have recently been applied to describe theoretically the thermal transport. In $[5,41])$, by applying the Boltzmann transport expressions for the thermal transport coefficients with numerical methods DFT and DFPT (see [5,41] and the the review [42] for references) the authors showed how one can combine the old well founded methods with the modern ones to depict graphically both the phonon and electron contribution to the thermal conductivity $[5,41]$ by first-principles calculations. In [41] these methods were applied to $\mathrm{NiAl}$ and $\mathrm{Ni}_{3} \mathrm{Al}$ and upgraded to certain noble and transition metals [5].

\subsection{Optical phonons}

The intermetallic compounds $\mathrm{REIn}_{3}$, which we examine, crystallize in the $\mathrm{AuCu}_{3}$ structure and have a basis of two atoms per unit cell. Thus, a single band of optical modes of the phonon spectrum can scatter valence electrons coming from two ions, $\mathrm{RE}^{+3}$ and $\mathrm{In}^{+3}$ ions, of the lattice. A precise determination of $\mathfrak{C}_{0}\left(\omega_{\mathbf{q}}, \mathbf{q}\right)$ of equation (3) for the scattering by optical phonons is in general a more difficult task than for acoustic ones since the problem of the electron-phonon interaction is more complicated (see, e.g. $[43,44]$ and references therein). However, we apply the simplest approximations in which the subtleties of the scattering described in $[43,44]$ will not be substantive. It is enough to mention that two types of electron-phonon interactions are known in the optical phonon case. The first is caused by a relative displacement of the sublattices and the resulting shift of the electron band edge. The second is a consequence of the electrical polarization induced by the optical phonons (see. e.g, [45] and references therein).

The first interaction apparently predominates in REIn 3 and is the only one which we take into account while considering these compounds. In order to describe the scattering of the conduction electrons by non-polar optic phonons it is formally sufficient to suppose that the squared matrix element responsible for the scattering, $\mathfrak{C}_{0}\left(\omega_{\mathbf{q}}, \mathbf{q}\right)$, is independent of $\mathbf{q}=\mathbf{k}^{\prime}-\mathbf{k}$. Then, the counterpart of (17) is obtained by replacing $\omega_{\mathbf{q}}$ by $\omega_{0}$ of (10) and $q=\left|\mathbf{k}^{\prime}-\mathbf{k}\right|$ by the magnitude of a primitive reciprocal lattice vector $G_{\mathrm{o}}$ [46]. In this way we obtain

$$
\mathfrak{C}_{0}\left(\omega_{\mathbf{q}}, \mathbf{q}\right)=\frac{V_{\mathrm{c}}}{4 \pi^{2}} \frac{E_{1}^{2} G_{\mathrm{o}}^{2}}{M \omega_{0}}
$$

being the standard formula used in theory of electronic transport in semiconductors [8]. It is well founded by the deformation potential methods [45]; the product $E_{1} G_{\mathrm{o}}$ is denoted as $D_{\mathrm{o}}$ and called the deformation potential constant. For our considerations the fact that $\mathfrak{C}_{0}$ can be assumed $q$-independent $(l=0$ in (13)) is of basic importance. The corresponding expression (17) applied to metals in case of acoustic phonons leads to the well founded temperature dependence for the electrical resistivity. Therefore, we take for granted that a q-independent magnitude of $\mathfrak{C}_{0}$ such as (25) is a proper first approximation also for metals.

In compounds such as $\mathrm{ReO}_{3}$ and $\mathrm{LaB}_{6}$ the electrical polarization effects are of basic importance and the polar electron-phonon interaction is considered to prevail over the non-polar one. This is because the configuration of the maximum charge distribution in these compounds is $\mathrm{Re}^{3+} \mathrm{O}^{-1}$ and $\mathrm{La}^{3+}\left(\mathrm{B}_{6}\right)^{2-}$, respectively, and therefore the ionic bonding occur. An experimental evidence for the influence of the polar optical modes in these compounds on the electrical resistivity was provided in [9-11]. A simple model of the scattering of the conduction electrons by polar phonon modes was presented by Fröhlich [18] who considered a crystal built of cells containing two ions of opposite electric charges of the masses $M^{+}$and $M^{-}$and the mutual distance $d$. Then, only longitudinal phonon waves contribute to the scattering probability (9) and the function $\mathfrak{C}_{0}\left(\omega_{\mathbf{q}}, \mathbf{q}\right)$ is following

$$
\mathfrak{C}_{0}\left(\omega_{\mathbf{q}}, \mathbf{q}\right) \equiv \frac{e^{4}}{\pi^{2} d^{3} q^{2} M^{\prime} \omega_{0}}
$$

where $-e$ denotes the electron charge and $1 / M^{\prime}=\left(1 / M^{+}\right)$ $+\left(1 / M^{-}\right)$. The exponent of $q$ in (13) being $l=-2$ in this case and will be essential for further considerations. From a more general standpoint than the Fröhlich model [18] 
this power is a consequence of calculating the gradient of the Fourier transform of the Coulomb potential in order to find the Fourier components of the polarization vector $[29,47]$ and the same power results from applying of the deformation potential approximation [45].

In both cases, (25) and (26), the limits of the integrals in (13) which follow from the energy conservation are following

$$
\begin{array}{ll}
\mathfrak{a}=\alpha\left[\epsilon^{1 / 2}-\epsilon_{+}^{1 / 2}\right], & \mathfrak{b}=\alpha\left[\epsilon^{1 / 2}+\epsilon_{+}^{1 / 2}\right], \\
\mathfrak{c}=\alpha\left[\epsilon^{1 / 2}-\epsilon_{-}^{1 / 2}\right], & \mathfrak{d}=\alpha\left[\epsilon^{1 / 2}+\epsilon_{-}^{1 / 2}\right],
\end{array}
$$

where $\alpha=(2 m / \hbar)^{1 / 2}, \epsilon_{+}=\epsilon+\hbar \omega_{0}$ and $\epsilon_{-}=\epsilon-\hbar \omega_{0}$. These limits should be used in case of semiconductors, as it was done in [12]. The assumption of the above upper limits $\mathfrak{b}, \mathfrak{d}$ in case of a metal would mean that a change of the electron wave number is about $2 k_{\mathrm{F}}$ for $\epsilon \approx \epsilon_{\mathrm{F}}$, whereas in fact it is less than the size of the Brillouin zone being smaller than $2 k_{\mathrm{F}}$. Therefore, while considering metals, we take also into account the upper limit of the phonon spectrum approximated by the magnitude of radius $q_{\mathrm{D}}$ of the Debye sphere. This is obviously the same assumption as for acoustic phonons, however, in the present case the magnitude of $q_{\mathrm{D}}$ has to be estimated by means of the (cubic) lattice constant for the considered crystal. In this way, we obtain the following $q_{\mathrm{D}}=\left(6 \pi^{2}\right)^{1 / 3} /(\gamma a)$ with $\gamma=1$ for the simple cubic lattice, $\gamma=(0.5)^{1 / 3}$ for bcc lattice, and $\gamma=(0.25)^{1 / 3}$ for fcc. Thus, by using this additional constraint for metals we assume that

$$
\mathfrak{a} \leq q \leq 2 q_{\mathrm{D}}, \quad \mathfrak{c} \leq q \leq 2 q_{\mathrm{D}},
$$

respectively, in case of the absorption and the emission, where $\mathfrak{a}$ i $\mathfrak{c}$ are given by equation (28) and it is not obvious that $\mathfrak{a}=\mathfrak{c}$ as in case of acoustic phonons. Thus, one cannot change the order of the integration in (12) as it is while considering acoustic phonons.

For convenience of the further considerations we introduce the dimensionless constant

$$
\xi=\frac{\hbar^{2}}{2 m} \frac{\left(6 \pi^{2}\right)^{2 / 3}}{\hbar \omega_{0} \gamma^{2} a^{2}} \approx \frac{2.31554 \cdot 10^{2} \mathrm{eV}}{\mu \gamma^{2} a_{\mathrm{A}}^{2} \hbar \omega_{0}}=\frac{2.68528 \cdot 10^{6} \mathrm{~K}}{\mu \gamma^{2} a_{\mathrm{A}}^{2} T_{\mathrm{E}}},
$$

where $m=\mu m_{\mathrm{o}}$ with $m_{\mathrm{o}}$ the mass of the free electron, $T_{\mathrm{E}}=\hbar \omega_{0} / k_{\mathrm{B}}$ is the Einstein temperature, and the lattice constant $a$ is represented by dimensionless quantity $a_{\mathrm{A}}$ $\left(a=a_{\mathrm{A}} \cdot 10^{-10} \mathrm{~m}\right)$, which in Angströms $(\AA)$ express its magnitude. By using this parameter one can introduce dimensionless functions $\Phi_{\mathrm{a}}(w), \Phi_{\mathrm{e}}(w)$ and $\Psi_{\mathrm{a}}(w), \Psi_{\mathrm{e}}(w)$ of the argument $w=\epsilon / \hbar \omega_{0}$, which differ for (25) and (26) and result from the integration of the right-hand side of (12) with respect $q$. Then the matrix elements (11) can be expressed in the following way

$$
\begin{aligned}
P_{11} / P_{\mathrm{o}}= & \mathcal{N}_{\mathrm{o}}^{\prime} \int_{0}^{\infty} \mathrm{d} \epsilon f_{0}(\epsilon)\left[1-f_{0}\left(\epsilon_{+}\right)\right] \Phi_{\mathrm{a}}(w) \\
& +\mathcal{N}_{\mathrm{o}}^{\prime \prime} \int_{\mathfrak{g}}^{\infty} \mathrm{d} \epsilon f_{0}(\epsilon)\left[1-f_{0}\left(\epsilon_{-}\right)\right] \Phi_{\mathrm{e}}(w)
\end{aligned}
$$

$$
\begin{aligned}
P_{12} / P_{\mathrm{o}}= & \mathcal{N}_{\circ}^{\prime} \int_{0}^{\infty} \mathrm{d} \epsilon f_{0}(\epsilon)\left[1-f_{0}\left(\epsilon_{+}\right)\right] u_{12}^{(\mathrm{a})} \Phi_{\mathrm{a}}(w) \\
& +\mathcal{N}_{\circ}^{\prime \prime} \int_{\mathfrak{g}}^{\infty} \mathrm{d} \epsilon f_{0}(\epsilon)\left[1-f_{0}\left(\epsilon_{-}\right)\right] u_{12}^{(\mathrm{e})} \Phi_{\mathrm{e}}(w)
\end{aligned}
$$

$P_{22} / P_{\mathrm{o}}$

$=\mathcal{N}_{\circ}^{\prime} \int_{0}^{\infty} \mathrm{d} \epsilon f_{0}(\epsilon)\left[1-f_{0}\left(\epsilon_{+}\right)\right]\left[u_{22}^{(\mathrm{a})} \Phi_{\mathrm{a}}(w)+\left(\hbar \omega_{0}\right)^{2} \Psi_{\mathrm{a}}(w)\right]$
$+\mathcal{N}_{\mathrm{o}}^{\prime \prime} \int_{\mathfrak{g}}^{\infty} \mathrm{d} \epsilon f_{0}(\epsilon)\left[1-f_{0}\left(\epsilon_{-}\right)\right]\left[u_{22}^{(\mathrm{e})} \Phi_{\mathrm{e}}(w)+\left(\hbar \omega_{0}\right)^{2} \Psi_{\mathrm{e}}(w)\right]$,

where $\mathcal{N}_{\mathrm{o}}^{\prime}=\mathcal{N}_{\mathrm{o}} / k_{\mathrm{B}} T, \mathcal{N}_{\mathrm{o}}^{\prime \prime}=\left(\mathcal{N}_{\mathrm{o}}+1\right) / k_{\mathrm{B}} T, \epsilon_{+}=\epsilon+\hbar \omega_{0}$, $\epsilon_{-}=\epsilon-\hbar \omega_{0}, \mathfrak{g}=\hbar \omega_{0}$ and the functions $u_{\mathrm{ij}}^{(\mathrm{a})}, u_{\mathrm{ij}}^{(\mathrm{e})}$ are defined

$$
\begin{aligned}
& u_{12}^{(\mathrm{a})}=(\epsilon-\zeta)+\hbar \omega_{0} \quad u_{12}^{(\mathrm{e})}=(\epsilon-\zeta)-\hbar \omega_{0} \\
& u_{22}^{(\mathrm{a})}=(\epsilon-\zeta)^{2}+2 \hbar \omega_{0}(\epsilon-\zeta)+\left(\hbar \omega_{0}\right)^{2} \\
& u_{22}^{(\mathrm{e})}=(\epsilon-\zeta)^{2}-2 \hbar \omega_{0}(\epsilon-\zeta)+\left(\hbar \omega_{0}\right)^{2}
\end{aligned}
$$

with $\mathcal{N}_{\mathrm{o}}=\left(\exp \left(\hbar \omega_{0} / k_{\mathrm{B}} T\right)-1\right)^{-1} . P_{\mathrm{o}}$ is certain material constant, the value of which is not essential for the present considerations. Explicit form the functions $\Phi_{\mathrm{a}}(w), \Psi_{\mathrm{a}}(w)$ and $\Phi_{\mathrm{e}}(w), \Psi_{\mathrm{e}}(w)$ will be presented in the following two subsections.

In order to estimate numerically the contribution of particular functions $\Phi_{\mathrm{a}}(w), \Phi_{\mathrm{e}}(w)$ and $\Psi_{\mathrm{a}}(w), \Psi_{\mathrm{e}}(w)$ to the matrix elements $P_{i j}$ we employ the identity (5), (6) and calculate the integrals of (31) in the lowest order of the Sommerfeld expansion. Let the matrix elements $P_{i j}$ approximated in this way are denoted $P_{i j}^{(\mathrm{s})}$ with $\mathcal{P}_{i j}(T)=\mathcal{P}_{i j}^{(\mathrm{s})}(T)=\mathcal{G}_{i j}^{(\mathrm{s})}\left(t_{\mathrm{E}}\right)$ as the corresponding functions of (1) and (4). The functions $\mathcal{G}_{i j}^{(\mathrm{s})}\left(t_{\mathrm{E}}\right)$ expressed by $p \equiv \hbar \omega_{0} / k_{\mathrm{B}} T=T_{\mathrm{E}} / T=t_{\mathrm{E}}^{-1}$ and the parameter $w_{\mathrm{F}}=$ $\epsilon_{\mathrm{F}} / \hbar \omega_{0}$ are

$$
\mathcal{G}_{11}^{(\mathrm{s})}\left(t_{\mathrm{E}}\right)=\frac{1}{\sinh (p)}\left[\Phi_{\mathrm{a}}\left(w_{\mathrm{F}}\right)+\Phi_{\mathrm{e}}\left(w_{\mathrm{F}}\right)\right],
$$

$$
\begin{aligned}
\mathcal{G}_{12}^{(\mathrm{s})}\left(t_{\mathrm{E}}\right)= & \frac{1}{\sinh (p)}\left\{p\left[\Phi_{\mathrm{a}}\left(w_{\mathrm{F}}\right)-\Phi_{\mathrm{e}}\left(w_{\mathrm{F}}\right)\right]\right. \\
& +\frac{\pi^{2}}{3} p\left[\Phi_{\mathrm{a}}^{\prime}\left(w_{\mathrm{F}}\right)+\Phi_{\mathrm{e}}^{\prime}\left(w_{\mathrm{F}}\right)\right] \\
& \left.-\frac{\pi^{2}}{6}\left[\Phi_{\mathrm{a}}\left(w_{\mathrm{F}}\right)-\Phi_{\mathrm{e}}\left(w_{\mathrm{F}}\right)\right] \tanh (p / 2)\right\}
\end{aligned}
$$




$$
\begin{aligned}
\mathcal{G}_{22}^{(\mathrm{s})}\left(t_{\mathrm{E}}\right)= & \frac{\pi^{2}}{3} \frac{1}{\sinh (p)}\left\{\Phi_{\mathrm{a}}\left(w_{\mathrm{F}}\right)+\Phi_{\mathrm{e}}\left(w_{\mathrm{F}}\right)\right. \\
& +2\left[\Phi_{\mathrm{a}}^{\prime}\left(w_{\mathrm{F}}\right)-\Phi_{\mathrm{e}}^{\prime}\left(w_{\mathrm{F}}\right)\right] \\
& -p\left[\Phi_{\mathrm{a}}\left(w_{\mathrm{F}}\right)+\Phi_{\mathrm{e}}\left(w_{\mathrm{F}}\right)\right] \tanh (p / 2) \\
& +\frac{3}{\pi^{2}} p^{2}\left[\Phi_{\mathrm{a}}\left(w_{\mathrm{F}}\right)+\Phi_{\mathrm{e}}\left(w_{\mathrm{F}}\right)\right. \\
& \left.\left.+\left[\Psi_{\mathrm{a}}\left(w_{\mathrm{F}}\right)+\Psi_{\mathrm{e}}\left(w_{\mathrm{F}}\right)\right]\right]\right\}
\end{aligned}
$$

The functions $\Phi_{\mathrm{a}}^{\prime}\left(w_{\mathrm{F}}\right)$ are $\Phi_{\mathrm{e}}^{\prime}\left(w_{\mathrm{F}}\right)$ in the above expressions are the derivatives of $\Phi_{\mathrm{a}}(w)$ and $\Phi_{\mathrm{e}}(w)$ with respect $w$ at $w=w_{\mathrm{F}}$. The values of the material constant $\mathcal{P}_{0}^{(\mathrm{op})}$, different for nonpolar and polar phonons are given in Appendix C. The interval of $w_{\mathrm{F}}$ in which equation (34) have a physical sense depends on the values of $T_{\mathrm{E}}, \epsilon_{\mathrm{F}}$ and the effective electron mass. The lower values of $\epsilon_{\mathrm{F}}$ are limited by the validity of the approximation $\zeta(T) \approx \epsilon_{\mathrm{F}}$ and the upper one by the number of the current carriers which may occur in metal. If one assumes a free electrons gas and $T_{\mathrm{E}} \approx 800 \mathrm{~K}$, the magnitude of $w_{\mathrm{F}}$ is only about $9 \lesssim w_{\mathrm{F}} \lesssim 40$, what corresponds to the values of $\epsilon_{\mathrm{F}}$ from the approximate interval $0.6 \mathrm{eV} \lesssim \epsilon_{\mathrm{F}} \lesssim 3 \mathrm{eV}$. The lower value in the latter inequalities follows from the requirement for the chemical potential $\zeta$ to be temperature independent (and equal to $\epsilon_{\mathrm{F}}$ ). The upper value of these inequalities is a consequence of the number of the conduction electrons in the crystal under consideration. For quasi-free electrons of the electron mass $m=\mu m_{\mathrm{o}}\left(m_{\mathrm{o}}\right.$ is the free electron mass) we have $\epsilon_{\mathrm{F}}=0.364675 \cdot 10^{-14} \mu^{-1} n_{\mathrm{e}}^{2 / 3} \mathrm{~cm}^{2} \mathrm{eV}$, which in case $n_{\mathrm{e}}=10^{22} \mathrm{~cm}^{-3}$ and $\mu=1$ yields $\epsilon_{\mathrm{F}} \approx 1.7 \mathrm{eV}$.

\subsubsection{Nonpolar optical phonons}

In case of nonpolar optical phonons, i.e., under the assumption (25), the functions $\Phi_{\mathrm{i}}(w) . \Psi_{\mathrm{i}}(w)(\mathrm{i}=\mathrm{a}, \mathrm{e})$ are following

$$
\Phi_{\mathrm{i}}(w)=\frac{1}{4}\left[\xi^{2}-\phi_{\mathrm{i}}^{4}(w)\right], \quad \Psi_{\mathrm{i}}(w)=\frac{1}{2} w\left[\xi-\phi_{\mathrm{i}}^{2}(w)\right]
$$

where

$$
\begin{aligned}
& \phi_{\mathrm{a}}(w)=(w+1)^{1 / 2}-w^{1 / 2}=\frac{1}{(w+1)^{1 / 2}+w^{1 / 2}} \\
& \phi_{\mathrm{e}}(w)=w^{1 / 2}-(w-1)^{1 / 2}=\frac{1}{w^{1 / 2}+(w-1)^{1 / 2}},
\end{aligned}
$$

with the values limited to $w>1$ for $\phi_{\mathrm{e}}(w)$.

Consider first the approximation (33-35). The numerical analysis of these formulas for (36) in the interval $9<w_{\mathrm{F}}<40$ indicates that an essential contribution to the functions $(33-35)$ comes from $\Phi_{\mathrm{i}}(w) \approx \xi^{2} / 4, \Psi_{\mathrm{i}}(w) \approx w \xi$, since the magnitude of the terms resulting from the functions $\phi_{\mathrm{a}}(w), \phi_{\mathrm{e}}(w)$ is several orders of the magnitude less. By assuming now that $\Phi_{\mathrm{i}}\left(w_{\mathrm{F}}\right)=\xi^{2} / 4, \Psi_{\mathrm{i}}\left(w_{\mathrm{F}}\right)=w_{\mathrm{F}} \xi$ and introducing these values to $(33-35)$ one can obtain simple formulas for the functions $\mathcal{G}_{11}^{(\mathrm{s})}\left(t_{\mathrm{E}}\right), \mathcal{G}_{12}^{(\mathrm{s})}\left(t_{\mathrm{E}}\right), \mathcal{G}_{22}^{(\mathrm{s})}\left(t_{\mathrm{E}}\right)$.
Notice that this assumption is equivalent to using the limits $\left(0,2 q_{\mathrm{D}}\right)$ of the integrals with respect $q$ instead of $\left(\mathfrak{a}, 2 q_{\mathrm{D}}\right)$ and $\left(\mathfrak{c}, 2 q_{\mathrm{D}}\right)$.

The assumption $\Phi_{\mathrm{i}}\left(w_{\mathrm{F}}\right)=\xi^{2} / 4, \Psi_{\mathrm{i}}\left(w_{\mathrm{F}}\right)=w_{\mathrm{F}} \xi$ at the level of the equations for (31) enables one to obtain also simple formulas $\mathcal{G}_{11}\left(t_{\mathrm{E}}\right), \mathcal{G}_{12}\left(t_{\mathrm{E}}\right), \mathcal{G}_{22}\left(t_{\mathrm{E}}\right)$ in a better approximation than the mentioned above. In this approximation the formulas for $\mathcal{G}_{i j}\left(t_{\mathrm{E}}\right)$ are counterparts of the Bloch-Grüneisen ones. As in the case of the acoustic phonons they are obtained by using the Wilson method [26] of integrating with respect the electron energy with taking into account the identity (5), (6). Several formula allowing to facilitate this calculation are presented in Appendix B. By employing these formula we obtain for the functions $\mathcal{P}_{i j}(T)=\mathcal{G}_{i j}\left(t_{\mathrm{E}}\right)$ of (1) and (4) the formulas

$$
\begin{aligned}
\mathcal{G}_{11}\left(t_{\mathrm{E}}\right) & =\frac{\xi^{2} p}{2\left(\mathrm{e}^{p}-1\right)\left(1-\mathrm{e}^{-p}\right)}=\frac{\xi^{2} p}{8 \sinh ^{2}(p / 2)} \\
\mathcal{G}_{22}\left(t_{\mathrm{E}}\right) & =\frac{p}{\left(\mathrm{e}^{p}-1\right)\left(1-\mathrm{e}^{-p}\right)}\left[\frac{1}{6} \xi^{2}\left(\pi^{2}+p^{2}\right)+\xi w_{\mathrm{F}} p^{2}\right] \\
& =\mathcal{G}_{11}\left(t_{\mathrm{E}}\right)\left[\frac{\pi^{2}}{3}+\left(\frac{1}{3}+\frac{2}{\xi} w_{\mathrm{F}}\right) p^{2}\right]
\end{aligned}
$$

and $\mathcal{G}_{12}\left(t_{\mathrm{E}}\right)=0$. The expressions for values of the relevant material constants $\rho_{0}^{(\mathrm{op})}$ and $\mathcal{P}_{\mathrm{o}}^{(\mathrm{op})}$ can be found in Appendix C.

\subsubsection{Polar optical phonons}

For polar optical phonons, it means under the assumption (26), the functions $\Phi_{\mathrm{i}}(w), \Psi_{\mathrm{i}}(w)$ (i=a,e) are

$$
\begin{aligned}
& \Phi_{\mathrm{i}}(w)=\xi-\phi_{\mathrm{i}}^{2}(w), \\
& \Psi_{\mathrm{i}}(w)=2 w \ln \left[\xi^{1 / 2} \phi_{\mathrm{i}}^{-1}\right]=w \ln \xi+2 w \ln \phi_{\mathrm{i}}(w) .
\end{aligned}
$$

The numerical analysis of (33), (35) such as in the previous Subsection allows to assume approximately $\Phi_{\mathrm{a}}(w)=$ $\Phi_{\mathrm{e}}(w)=\xi$ but the functions $\Psi_{\mathrm{a}}(w), \Psi_{\mathrm{e}}(w)$ - unlike their counterparts in the previous Subsection - have to be taken into account without any approximation. By assuming $\Phi_{\mathrm{a}}(w)=\Phi_{\mathrm{e}}(w)=\xi$ in $(33)-(35)$ one can represent the matrix elements $\mathcal{P}_{i j}(T)$ of (4) by simple formulas corresponding to the Sommerfeld approximation.

In much the same way as in the case of (38), (39), one can obtain formulas corresponding to the BlochGrüneisen functions of the scattering by acoustic phonons. Appendix B facilitates to pass the calculations. The final results for the matrix elements $\mathcal{P}_{i j}(T)=\mathcal{G}_{i j}\left(t_{\mathrm{E}}\right)$ are

$$
\begin{aligned}
\mathcal{G}_{11}\left(t_{\mathrm{E}}\right) & =\frac{2 \xi p}{\left(\mathrm{e}^{p}-1\right)\left(1-\mathrm{e}^{-p}\right)}=\frac{\xi p}{2 \sinh ^{2}(p / 2)}, \\
\mathcal{G}_{12}\left(t_{\mathrm{E}}\right)= & 0 \\
\mathcal{G}_{22}\left(t_{\mathrm{E}}\right)= & \frac{2 \xi p\left(\pi^{2}+p^{2}\right)}{3\left(\mathrm{e}^{p}-1\right)\left(1-\mathrm{e}^{-p}\right)} \\
& +\frac{2 p^{3}}{\left(\mathrm{e}^{p}-1\right)\left(1-\mathrm{e}^{-p}\right)}\left[w_{\mathrm{F}} \ln \xi+\Lambda\left(w_{\mathrm{F}}, p\right)\right] \\
= & \mathcal{G}_{11}\left(t_{\mathrm{E}}\right)\left\{\pi^{2}+p^{2}\left[w_{\mathrm{F}} \ln \xi+\Lambda\left(w_{\mathrm{F}}, p\right)\right]\right\}
\end{aligned}
$$


where $p=\hbar \omega_{0} / k_{\mathrm{B}} T=T_{\mathrm{E}} / T=t_{\mathrm{E}}^{-1}$, and the function $\Lambda\left(w_{\mathrm{F}}, p\right)$ is defined

$$
\begin{aligned}
\Lambda\left(w_{\mathrm{F}}, p\right)= & \frac{1}{p} \int_{0}^{p} \mathrm{~d} y(2 w-1) \ln \left[w^{1 / 2}+(w-1)^{1 / 2}\right] \\
\approx & w_{\mathrm{F}}\left\{\ln w_{\mathrm{F}}^{1 / 2}+\left(w_{\mathrm{F}}+1\right)^{1 / 2}\right] \\
& \left.+\ln \left[w_{\mathrm{F}}^{1 / 2}+\left(w_{\mathrm{F}}-1\right)^{1 / 2}\right]\right\}
\end{aligned}
$$

The variables in the above formula are $w=p^{-1}(y+z) \approx$ $w_{\mathrm{F}}+y / p, z=\zeta(T) / k_{\mathrm{B}} T \approx \epsilon_{\mathrm{F}} / k_{\mathrm{B}} T$. The relevant material constants are denoted in the way as in the previous Subsection, namely $\rho_{0}^{(\mathrm{op})}$ and $\mathcal{P}_{\mathrm{o}}^{(\mathrm{op})}$, and the appropriate expressions can be found in Appendix C.

Much the same formula as our $\mathcal{G}_{11}\left(t_{\mathrm{E}}\right)$, (41), was obtained by Howarth and Sondheimer [12] as a limiting case for the degenerate electron gas in their considerations of the influence of the conduction electron scattering on the electrical resistivity in polar semiconductors. The difference is in the factor $\xi$, being a consequence of the fact that unlike those of [12] our integrals with respect $q$ are limited to the Debye sphere. The formula for the degenerate gas of $[12]$ was applied in $[9,10]$ to improve the fitting of the experimental electrical resistivity data of $\mathrm{ReO}_{3}$ and $\mathrm{LaB}_{6}$ to theoretical curves. In the present paper we extend the consideration such as applied in $[9,10]$ to the thermal resistivity. According to our knowledge there is no such extension neither in the case of scattering by polar nor nonpolar phonons.

\section{Scattering of conduction electrons by localized magnetic moments}

In order to find the form of $\mathfrak{C}(\mathbf{k}, \mathbf{q})$ of (3) in the case of magnetic scattering we assume the conduction electron spins to interact via the exchange interaction with the spins of $4 \mathrm{f}$ ions being under influence of a crystal field and a Heisenberg-like interaction between the ions. Let the local interaction of a single conduction electron spin $\mathbf{s}$ with the total spin $\mathbf{S}$ of the electronic shell of a $\mathrm{RE}$ ion is $j_{\mathrm{ex}} \mathbf{S} \mathbf{S},[1,48,49]$, where the exchange energy is of the order of a few tenth of eV. For further convenience we shall represent this interaction in terms of the total stable magnetic moment of the $4 \mathrm{f}$-shell $\mathbf{J}$, it means as $j_{\mathrm{ex}}(g-1) \mathbf{s} \mathbf{J}$, where $g$ is the Lande factor. Consider now the whole crystal of the volume $V$ with $N_{4 \mathrm{f}} \mathrm{RE}$ ions. The total exchange energy, $\mathcal{H}_{\mathrm{s}-\mathrm{f}}$, of the interaction of the conduction electrons with $4 \mathrm{f}$ ions ( $\mathrm{s}-\mathrm{f}$ interaction) is assumed to be sufficiently small in comparison the sum of the crystal field one and the Heisenberg-like one, $\mathcal{H}_{4 \mathrm{f}}$, so as it can be treated as a perturbation. For the system $\mathcal{H}_{4 \mathrm{f}}+\mathcal{H}_{\mathrm{s}-\mathrm{f}}$ one can find the transition probability $\mathfrak{C}(\mathbf{k}, \mathbf{q})$ calculated in the first Born approximation. As in the previous scattering systems of the present paper this transition probability calculated in this approximation can be considered to be sufficient for examining the transport properties. It can be represented by a simple, compact form which was found in
[50] in which $\mathfrak{C}(\mathbf{k}, \mathbf{q}) \equiv \mathfrak{C}(\Omega, \mathbf{q})$ is explicitly dependent on $\hbar \Omega \equiv \epsilon^{\prime}-\epsilon=\epsilon\left(\mathbf{k}^{\prime}\right)-\epsilon(\mathbf{k})$ and $\mathbf{q}$ in the following way

$$
\begin{aligned}
\mathfrak{C}(\Omega, \mathbf{q})= & \frac{V}{N_{4 \mathrm{f}}} \frac{j_{\text {ex }}^{2}(g-1)^{2}}{(2 \pi)^{3} \hbar} \mathfrak{C}^{(\mathrm{sf})}(\Omega, \mathbf{q}), \\
\mathfrak{C}^{(\mathrm{sf})}(\Omega, \mathbf{q})= & \frac{1}{N_{4 \mathrm{f}}} \sum_{n} \mathrm{e}^{-\mathbf{i} \mathbf{q}\left(\mathbf{R}_{n}-\mathbf{R}_{0}\right)} \\
& \times \int_{-\infty}^{\infty} \mathrm{d} t \mathrm{e}^{\mathrm{i} \Omega t}\left\langle\mathbf{J}_{n}(t) \mathbf{J}_{0}(\mathbf{0})\right\rangle_{4 \mathrm{f}},
\end{aligned}
$$

where $\mathbf{q}=\mathbf{k}^{\prime}-\mathbf{k}, \mathbf{R}_{n}-\mathbf{R}_{0}$ denotes the location of the moment $\mathbf{J}_{\mathbf{n}}$ in the lattice with respect to $\mathbf{J}_{0}$. The sum is taken over all the lattice points (including $n=0$ ) in the volume $V$ of the crystal. $\mathfrak{C}^{(\text {sf })}(\Omega, \mathbf{q})$ is obviously spacetime Fourier transform of the site-site correlation function in which $\mathbf{J}_{n}(t)=\exp \left(\mathrm{i} \mathcal{H}_{4 \mathrm{f}} t / \hbar\right) \mathbf{J}_{n}(0) \exp \left(-\mathrm{i} \mathcal{H}_{4 \mathrm{f}} t / \hbar\right)$ and the mean value $\langle\cdots\rangle_{4 \mathrm{f}}$ is calculated with $\mathcal{H}_{4 \mathrm{f}}$. The above transition probability resembles the expression for the neutron cross-section scattering in magnetic materials [20]. In both cases the scattering is described by similar correlation function.

To perform calculations in which we find the scattering matrices $P_{i j}$, (11), we make further assumption. We restrict our considerations to RE ions periodically ordered in the lattice. Moreover, for illustration, we assume the Heisenberg-like interaction leading to ferromagnetic ordering between $\mathrm{RE}$ ions at sufficiently low temperatures. In a further step we simplify this interaction by applying the molecular field approximation (MFA). Taking antiferromagnetic interaction into account within this approximation causes solely computational difficulties. After applying the mentioned model and simplifications, the Hamiltonian $\mathcal{H}_{4 \mathrm{f}}=\sum_{n} \mathcal{H}_{n}^{(1)}$ is a sum of the single-site independent Hamiltonians $\mathcal{H}_{n}^{(1)}$ which can be written

$$
\mathcal{H}_{n}^{(1)} \equiv \mathcal{H}=\mathcal{H}_{\mathrm{CF}}-\lambda \sigma J^{z}+\frac{1}{2} \lambda \sigma^{2}
$$

The first term $\mathcal{H}_{\mathrm{CF}}$ denotes the crystal field Hamiltonian (see Sect. 6). The coefficient $\lambda$ in (45) stands for the molecular field constant which can be expressed by an appropriate sum of the two-ion coupling constants. Without loss of the generality we can assume that the reduced spontaneous magnetization is $\langle\mathbf{J}\rangle=(0,0, \sigma)$. The magnitude of $\sigma=\sigma(T)$ has to be calculated in a self-consistent way by solving the equation $\sigma=Z^{-1} \operatorname{Tr} J^{z} \mathrm{e}^{-\mathcal{H} / k_{\mathrm{B}} T}$, where $Z=\operatorname{Tre}^{-\mathcal{H} / k_{\mathrm{B}} T}$.

Notice that we neglect the splitting of the conduction band under influence of the internal (molecular) magnetic field, which is an essential assumption of early papers [48, 49]. As it is known now this splitting in the ordered phase is only important in d-electron metals.

In order to calculate $\mathfrak{C}^{(\mathrm{sf})}(\Omega, \mathbf{q})$ we take for granted that the eigenvalues and eigenfunctions of the Hamiltonian $\mathcal{H}_{n}^{(1)}$ are known and denote them $E_{\alpha},\left|E_{\alpha}\right\rangle$, respectively. 
In this way in MFA we obtain

$$
\begin{aligned}
\mathfrak{C}^{(\mathrm{sf})}(\Omega, \mathbf{q}) \approx & \mathfrak{C}_{\mathrm{MFA}}^{(\mathrm{sf})}(\Omega, 0) \\
= & \frac{2 \pi}{Z} \sum_{\alpha, \beta=1}^{2 J+1} \mathrm{e}^{-E_{\alpha} / k_{\mathrm{B}} T} \mathbb{Q}_{\alpha \beta} \delta\left(\hbar \Omega-E_{\alpha}+E_{\beta}\right) \\
& -2 \pi\langle\mathbf{J}\rangle^{2} \delta(\hbar \Omega)
\end{aligned}
$$

where the states are numbered from 1 to $2 J+1$ and $\mathbb{Q}_{\alpha \beta}=$ $\mathbb{Q}_{\alpha \beta}$ are the elements of the matrix denoted $\mathbb{Q}$ which are

$$
\begin{aligned}
\mathbb{Q}_{\alpha \beta}= & \left|\left\langle E_{\alpha}\left|J^{z}\right| E_{\beta}\right\rangle\right|^{2} \\
& +\frac{1}{2}\left|\left\langle E_{\alpha}\left|J^{+}\right| E_{\beta}\right\rangle\right|^{2}+\frac{1}{2}\left|\left\langle E_{\alpha}\left|J^{-}\right| E_{\beta}\right\rangle\right|^{2},
\end{aligned}
$$

where the standard notation is used for the components $J^{+}, J^{-}$and $J^{z}$ of $\mathbf{J}_{\mathbf{0}} \equiv \mathbf{J}, \quad Z=$ $\sum_{\alpha} \exp \left(-E_{\alpha} / k_{\mathrm{B}} T\right)$ is the partition function and $\langle\mathbf{J}\rangle=$ $Z^{-1} \sum_{\alpha} \exp \left(-E_{\alpha} / k_{\mathrm{B}} T\right)\left\langle E_{\alpha}|\mathbf{J}| E_{\alpha}\right\rangle$. The summation in (47) concerns the pairs $\alpha \beta$ and $\beta \alpha$ when $\alpha \neq \beta$. For the degenerated states we assume $\alpha=\beta$ and then $\Omega=0$.

The independence $\mathfrak{C}^{(\mathrm{sf})}(\Omega, \mathbf{q})$ of $\mathbf{q}$ permit us to calculate easily the integrals (2) with respect the angles of the spherical coordinates of the vectors $\mathbf{k} \mathbf{i} \mathbf{k}^{\prime}$. Therefore, the matrix element $P_{i j}$, equation (2), can be expressed in terms of the products of $Q_{\alpha, \beta}$ and and integrals with respect $\epsilon \mathrm{i} \epsilon^{\prime}$ in the limits $(0, \infty)$ of functions of the structure

$$
\mathcal{K}_{i j}=\left(-\frac{\partial f_{0}}{\partial \epsilon}\right) \mathfrak{G}\left(\epsilon, \epsilon^{\prime}\right) U_{i j} \delta\left(\epsilon-\epsilon^{\prime}-\Delta_{\alpha \beta}\right)
$$

where $\Delta_{\alpha \beta}=E_{\alpha}-E_{\beta}, U_{i j}$ are simple polynomials of $\epsilon, \epsilon^{\prime}$ and $\epsilon-\zeta, \epsilon^{\prime}-\zeta$, and the function $\mathfrak{G}\left(\epsilon, \epsilon^{\prime}\right)$ is defined by (6). The final expressions in the metallic limit are obtained by applying the Sommerfeld expansion for the mentioned integrals. The functions $\mathcal{P}_{i j}(T)$ of (4) in this case are

$$
\begin{aligned}
& \mathcal{P}_{11}(T) \equiv \mathcal{R}_{\mathrm{J}}(T)=\operatorname{Tr} \mathbb{R} \mathbb{Q}-\langle\mathbf{J}\rangle^{2} \\
& \mathcal{P}_{12}(T)=2 \frac{\pi^{2}}{3}\left(k_{\mathrm{B}} T\right) \frac{k_{\mathrm{B}} T}{\epsilon_{\mathrm{F}}} \mathcal{R}_{\mathrm{J}}(T) \\
& \mathcal{P}_{22}(T) \equiv \mathcal{W}_{\mathrm{J}}(T)=\operatorname{Tr} \mathbb{W} \mathbb{Q}-\left(\pi^{2} / 3\right)\langle\mathbf{J}\rangle^{2},
\end{aligned}
$$

where $\mathbb{R}, \mathbb{W}$ are symmetric matrices the elements

$$
\begin{aligned}
\mathbb{R}_{\alpha \beta}= & \frac{2}{Z_{\mathcal{E}}} \frac{1}{\mathrm{e}^{+\mathcal{E}_{\alpha} / k_{\mathrm{B}} T}+\mathrm{e}^{+\mathcal{E}_{\beta} / k_{\mathrm{B}} T}} \\
\mathbb{W}_{\alpha \beta}= & \frac{1}{Z_{\mathcal{E}}} \frac{1}{\mathrm{e}^{+\mathcal{E}_{\alpha} / k_{\mathrm{B}} T}+\mathrm{e}^{+\mathcal{E}_{\beta} / k_{\mathrm{B}} T}} \\
& \times\left[2 \frac{\pi^{2}}{3}-\frac{\pi^{2}}{3} \frac{\Delta_{\alpha \beta}}{k_{\mathrm{B}} T} \tanh \left(\frac{\Delta_{\alpha \beta}}{2 k_{\mathrm{B}} T}\right)+\left(\frac{\Delta_{\alpha \beta}}{k_{\mathrm{B}} T}\right)^{2}\right]
\end{aligned}
$$

The quantities $\mathcal{E}_{\alpha}=E_{\alpha}-E_{1}, \mathcal{E}_{\beta}=E_{\beta}-E_{1}$ denote the excitations $\Delta_{\alpha \beta}=E_{\alpha}-E_{\beta}=\mathcal{E}_{\alpha}-\mathcal{E}_{\beta}$ over the ground state $E_{1}, Z_{\mathcal{E}}=\sum_{\alpha} \exp \left(-\mathcal{E}_{\alpha} / k_{\mathrm{B}} T\right)$. The relevant material constant $\mathcal{P}_{\mathrm{O}}$ of (4) denoted as $\mathcal{P}_{\mathrm{o}}^{\text {(sf) }}$ is the same as its counterpart in [27] and is presented in Appendix C.

Similar formula for the magnetic part of the electrical resistivity in the paramagnetic phase to that described by (49) with the matrix elements (50) is often applied as a test of the influence of crystal field splitting on the temperature dependence of the electrical resistivity. The formula was presented for the first time in [51] with its derivation presented is [52]. In our notations, the matrix elements of $\mathbb{R}_{\alpha \beta}$ of $[51,52]$ are

$$
\mathbb{R}_{\alpha \beta}=\left\{\begin{array}{l}
\left(p_{\alpha} \Delta_{\alpha \beta} / k_{\mathrm{B}} T\right)\left[1-\mathrm{e}^{-\Delta_{\alpha \beta} / k_{\mathrm{B}} T}\right]^{-1} \\
p_{\alpha} \delta_{\alpha \beta}
\end{array}\right.
$$

where the upper case corresponds to $\Delta_{\alpha \beta} \neq 0$, the lower one to $\Delta_{\alpha \beta}=0$ and $p_{\alpha}=\mathrm{e}^{-E_{\alpha} / k_{\mathrm{B}} T} / \sum_{\alpha} \mathrm{e}^{-E_{\alpha} / k_{\mathrm{B}} T}$ is the population of the $\alpha$-th state. Discrepancies between the results of (52) and (50) are only expected at an intermediate temperature region. At low temperatures and high ones both the formulas yield values close to 0 and $J(J+1)$, respectively. Notice that our derivation is more general since - as it follows from [52] - the formula (52) is obtained from (44) by representing the correlation function by the generalized susceptibility, and then by expanding the susceptibility in terms of the effective field strength with rejecting higher order terms than linear ones.

There is a very limited amount of cubic intermetallic RE compounds of ferromagnetic order. Therefore, the term $\langle\mathbf{J}\rangle^{2}$ in (49) is rather an illustration of the way in which the magnetic ordering manifest itself in the expressions for the electrical and thermal resistivity. Antiferromagnetic ordering, characteristic of these compounds, require special consideration. In our analysis of experimental results we confine to considering (49) in paramagnetic phases it means the cases when $\langle\mathbf{J}\rangle^{2}=0$ in (49).

An example of cubic intermetallic RE compounds of ferromagnetic order is $\mathrm{GdAl}_{2}$. Since the orbital magnetic moment of the Gd ions is equal to zero, the crystal field does not influence the behavior of $\mathrm{GdAl}_{2}$ and we have $\mathcal{H}_{\mathrm{CF}}$ in (45). The Curie temperature, $T_{\mathrm{c}}$, of this compound is rather high (about $150 \mathrm{~K}$ ), therefore the term $\langle\mathbf{J}\rangle^{2}$ contribute to (49) in a considerable temperature region. The formulas (49) are still valid in the case $\mathcal{H}_{\mathrm{CF}}=0$ and correspond to those obtained in [27] and corrected in the Appendix of [53].

To show this correspondence we consider first the paramagnetic phase, $T>T_{\mathrm{c}}$, of a crystal in which $\mathcal{H}_{\mathrm{CF}}=0$. Then $\langle\mathbf{J}\rangle^{2}=\sigma^{2}=0, \mathcal{E}_{\alpha}=\mathcal{E}_{\beta}=\Delta_{\alpha \beta}=0$ and therefore we have an elastic electron scattering which implies temperature independent values $\mathcal{R}_{\mathrm{J}}(T)=J(J+1), \mathcal{W}_{\mathrm{J}}(T)=$ $\left(\pi^{2} / 3\right) J(J+1)$. The inelastic scattering below $T_{\mathrm{c}}$ is a consequence of the excitations of the RE ions in the molecular field $\Delta_{\alpha, \beta}= \pm \lambda \sigma$ contributing if $\alpha=\beta+1$ or $\alpha=\beta-1$ in the second and the third terms of (47). These terms are completed with the "elastic" ones $\Delta_{\alpha, \beta}=0$ corresponding to $\alpha=\beta$ of the first term in (47). This simple excitation scheme allows to represent $\operatorname{Tr} \mathbb{R} \mathbb{Q}$ and $\operatorname{Tr} \mathbb{W} \mathbb{Q}$ in (49) in terms of $\left\langle J^{+} J^{-}\right\rangle=2 \sigma /\left(\mathrm{e}^{x}-1\right),\left\langle J^{-} J^{+}\left\langle=2 \sigma /\left(1-\mathrm{e}^{-x}\right)\right.\right.$ and $\left\langle\left(J^{z}\right)^{2}\left\langle=J(J+1)-\sigma \operatorname{coth} x\right.\right.$, where $x=\lambda \sigma /\left(k_{\mathrm{B}} T\right)$ and the averages $\langle\cdots<$ are calculated with the application of the molecular field Hamilton (45) with $\mathcal{H}_{\mathrm{CF}}=$ 0 . The expressions for the counterparts of $\mathcal{R}_{\mathrm{J}}(T)$ and $\mathcal{W}_{\mathrm{J}}(T)$ in [27] are also composed of these averages. The final expressions for these functions following from (49) 
and the considerations of [27] are the same and with the correction of $\mathcal{W}_{\mathrm{J}}(T)$ in the Appendix of [53] can be represented as

$$
\begin{gathered}
\mathcal{R}_{\mathrm{J}}(T)=J(J+1)-\sigma^{2}-\sigma \tanh (x / 2) \\
\mathcal{W}_{\mathrm{J}}(T)=\frac{\pi^{2}}{3} \mathcal{R}_{\mathrm{J}}(T)-\frac{\pi^{2}}{3} \frac{\sigma x}{2(\cosh x+1)}+\frac{\sigma x^{2}}{\sinh x} .
\end{gathered}
$$

The second and third term in (54) come from the summation of the second and third term of (51), respectively. The above functions depend, in fact, on the ratio $T / T_{\mathrm{c}}$ since the Curie temperature $T_{\mathrm{c}}$ is proportional to the molecular field strength constant $\lambda$ and the magnitude of $\sigma$, being the solution of the equation including the Brillouin function (see, e.g. [27]), is also a function of $T / T_{\mathrm{c}}$ with the value $\sigma=0$ for $T \geq T_{\text {c }}$.

\section{Lorenz number and the magnitude of the $s-f$ contribution to the electrical and thermal resistivity at high temperatures}

The temperature dependence of the Lorenz number $\mathcal{L}(T)=\rho(T) / T W(T)$ helps in an analysis of experimental data. Thus, consider also $\mathcal{L}$ for our model, it means the ratio $\rho(T)$ and $T W(T)$ with (1) and the particular functions given in Sections 3 and 4. If inelastic scattering is not taken into account within the models of these sections, we obtain $\mathcal{F}_{22}\left(t_{\mathrm{D}}\right)=\frac{\pi^{2}}{3} \mathcal{F}_{11}\left(t_{\mathrm{D}}\right), \mathcal{G}_{22}\left(t_{\mathrm{E}}\right)=\frac{\pi^{2}}{3} \mathcal{G}_{11}\left(t_{\mathrm{E}}\right)$ and $\mathcal{W}_{\mathrm{J}}(T)=\frac{\pi^{2}}{3} \mathcal{R}_{\mathrm{J}}(T)$ what imply that $\mathcal{L}(T)=\mathcal{L}_{\mathrm{O}}$. It means that the departure from the value of $\mathcal{L}_{\mathrm{o}}$ in $\mathcal{L}(T)$ is caused by the inelastic scattering.

The influence of the inelastic scattering diminishes over an increase of the temperature. In the limit $T \rightarrow \infty$ the functions $\rho$ and $W(T)$ are comprised of can be represented at high temperatures by asymptotic expressions: $\mathcal{F}_{11}\left(t_{\mathrm{D}}\right) \approx \frac{1}{4} t_{\mathrm{D}}, \mathcal{F}_{22}\left(t_{\mathrm{D}}\right) \approx \frac{1}{4} \frac{\pi^{2}}{3} t_{\mathrm{D}}, \mathcal{G}_{11}\left(t_{\mathrm{E}}\right) \approx g t_{\mathrm{E}}^{-1}$, $\mathcal{G}_{22}\left(t_{\mathrm{E}}\right) \approx g \frac{\pi^{2}}{3} t_{\mathrm{E}}^{-1}$ with with $g_{1}=\xi^{2} / 2$ in case of nonpolar optical phonons and $g=2 / \xi$ in case of polar optical phonons. The asymptotic expressions for the functions describing the $\mathrm{s}-\mathrm{f}$ scattering in case $T \rightarrow \infty$ are: $\mathcal{R}_{\mathrm{J}}(T) \approx J(J+1)+r /\left(k_{\mathrm{B}} T\right)$ and $\mathcal{W}_{\mathrm{J}}(T) \approx \frac{\pi^{2}}{3} J(J+$ 1) $+\frac{\pi^{2}}{3} r /\left(k_{\mathrm{B}} T\right)$, where $r$ is certain constant which value is not essential. By applying the above asymptotic expressions to that for $\mathcal{L}(T)$ one can easily find that $\lim _{T \rightarrow \infty} \mathcal{L}(T)=\mathcal{L}_{\mathrm{o}}$.

The asymptotic values of $\mathcal{R}_{\mathrm{J}}(T)$ and $\mathcal{W}_{\mathrm{J}}(T)$ at high temperatures also allow to write down a simple formulas for the "magnetic part" of the electrical resistivity called "the spin-disorder resistivity" $\lim _{T \rightarrow \infty} \rho_{\mathrm{o}}^{\text {(sf) }} \mathcal{R}_{\mathrm{J}}(T)$ and its counterpart of the thermal resistivity, being equal in our notation to $\lim _{T \rightarrow \infty}\left(\rho_{\mathrm{o}}^{\text {(sf) }} / \mathcal{L}_{\mathrm{O}} T\right) \mathcal{W}_{\mathrm{J}}(T)$. The latter quantity was used by the authors of $[6,16]$ (see Eqs. (7) and (8) in [6]) to examine the experimental data. By applying the above asymptotic value and the explicit expression for $\rho_{\mathrm{o}}^{\text {(sf) }}$ (see Appendix C) one obtains

$$
\begin{aligned}
\lim _{T \rightarrow \infty} \frac{\rho_{\mathrm{o}}^{(\mathrm{sf})}}{\mathcal{L}_{\mathrm{o}} T} \mathcal{W}_{\mathrm{J}}(T) & \equiv \frac{\rho_{\mathrm{o}}^{(\mathrm{sf})}}{\mathcal{L}_{\mathrm{o}} T(g-1)^{2}} \mathcal{W}_{\mathrm{J}}(T) \\
& \approx(g-1)^{2} J(J+1) \frac{C}{T},
\end{aligned}
$$

where the magnitude of $C$ can be easily found from the expression (C.8) of Appendix C. The slope of the spin disorder thermal resistivity as a function of $1 / T$ can be found from experimental measurements (see Fig. 2 in [6]).

\section{Phenomenological analysis of experimental data}

In order to demonstrate how the final formulas of the described theoretical method correspond to the measured electrical and thermal resistivity of real materials we apply them to the data of two mono-crystal intermetallic compounds, namely $\mathrm{DyIn}_{3}$ and $\mathrm{TmIn}_{3}$. The data for the mentioned crystals come from the same source [6]; the author of [6] measured both the thermal conductivity and electrical resistivity by using the same sample. Of the $\mathrm{REIn}_{3}$ series investigated in [6] we chose these two since they seem to most resemble the so-called "normal metals" as to the Fermi surface and the localization of the uf electrons. Some complications are caused by their antiferromagnetic ordering at low temperatures. Fortunately, their Néel temperatures are rather low as given in the Introduction, especially for $\mathrm{TmIn}_{3}$. We confine our analysis to temperatures above these temperatures to avoid a discussion of the magnetic fluctuations which are not included in our theoretical considerations.

As it is seen from equations (49)-(51), the ground multiplet of the localized electron subsystem has a real impact on the s-f scattering as well as its splitting and excited eigenstates. The determination of the s-f contribution to the considered coefficients requires the knowledge of the eigenstates and eigenvalues $\left|E_{\alpha}\right\rangle, E_{\alpha}$, of this subsystem (see Sect. 4).

The underlying eigenvalue problem is solved by simultaneous diagonalization of the internal Coulomb and spin-orbit interactions with the crystalline field potential. The Slater integrals and the spin-orbit coupling constants can be such as reported in [54] for the nonmetallic series $\mathrm{REF}_{3}$ Consequently, it is required and most convenient to use the basic Wybourne parameterization for the CF Hamiltonian [55]. It allows one not to be limited to the Russell-Sanders coupling and, furthermore, to control appropriate parameters in a series of compounds such as REIn 3 since their values should not vary too much for subsequent compounds of the series. More precisely, the absolute values are expected to monotonically decrease with increasing atomic number of the f-electron ion. For the needs of the present calculations we assume linear dependence on the lattice constant of the CF parameters $B_{40}$ and $B_{60}$ in the following crystal 
Table 1. Lattice constants and crystalline field parameters used to determine the s-f contribution to the electrical and thermal resistivity.

\begin{tabular}{lccc}
\hline & $\begin{array}{c}\text { Latt. const. } \\
(\AA)\end{array}$ & $\begin{array}{c}B_{40} \\
(\mathrm{~K})\end{array}$ & $B_{60}$ \\
& 4.5806 & 299 & 263 \\
$\mathrm{DyIn}_{3}$ & 4.5580 & 249 & 50 \\
$\mathrm{TmIn}_{3}$ & &
\end{tabular}

field Hamiltonian

$$
\begin{aligned}
\mathcal{H}_{\mathrm{CF}}= & \sum_{i}\left\{B_{40}\left(\hat{C}_{0}^{(4)}\left(\mathbf{r}_{i} / r_{i}\right)+\left(\frac{5}{14}\right)^{1 / 2} \hat{C}_{4}^{(4)}\left(\mathbf{r}_{i} / r_{i}\right)\right)\right. \\
& \left.+B_{60}\left(\hat{C}_{0}^{(6)}\left(\mathbf{r}_{i} / r_{i}\right)-\left(\frac{7}{2}\right)^{1 / 2} \hat{C}_{4}^{(6)}\left(\mathbf{r}_{i} / r_{i}\right)\right)\right\}(56)
\end{aligned}
$$

in which $i$ runs over all unpaired electrons of the unfilled shell of the metal ion, and $\hat{C}_{m}^{(n)}$ denote $m$ component of spherical tensor operator of rank $n$. When this Hamiltonian is introduced into (45), it determines together with the molecular field terms (if the system in an ordered phase) the quantum states of the localized electron subsystem involved in the considered scattering process.

For metallic systems, the determination of the $\mathrm{CF}$ parameters $B_{n m}$ is usually encumbered with considerable uncertainty. While looking through the values of the $\mathrm{CF}$ parameters available for the whole $\mathrm{REIn}_{3}$ series from the literature we found that in [56] the leading $\mathrm{B}_{40}$ parameter for $\mathrm{DyIn}_{3}$ is about six times lower than that for the remaining members of the series. Although we are not sure if the available data for the whole series are definitely reliable, we decided to assume interpolated values of $\mathrm{B}_{40}$ out of those represented as a function of the lattice con-

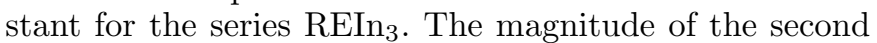
parameter $\mathrm{B}_{60}$ for $\mathrm{DyIn}_{3}$ was obtained in such a way to match the inelastic neutron scattering transition observed at $16.7 \mathrm{~K}$ for this compound [56]. In the case of $\mathrm{TmIn}_{3}$ both the CF parameters were determined by the mentioned above interpolation since the available estimations [57] are highly inconsistent with values obtained for other compounds of the series even as to the sign. Therefore, they were not taken into account by us. Obviously, the interpolated values shown in Table 1 , which are accepted for the present modeling, require confirmation by further specific material investigations.

Figure 1 shows the CF splittings of the ground J manifolds, ${ }^{6} \mathrm{H}_{15 / 2}$ and ${ }^{3} \mathrm{H}_{6}$ which correspond to the $4 \mathrm{f}^{9}$ (Dy) and $4 \mathrm{f}^{12}$ (Tm) configurations. The magnitude of the splitting was determined by using the values of the crystal field parameters obtained in the above described way.

As one could expect, the splitting for $\mathrm{DyIn}_{3}$ is larger than for $\mathrm{TmIn}_{3}$. This fact has consequences in qualitatively different influence of the s-f scattering. In particular it translates into a greater temperature interval in which the s-f scattering essentially influences the electrical and thermal resistivities. Above this interval the s-f mechanism produces a temperature independent component just

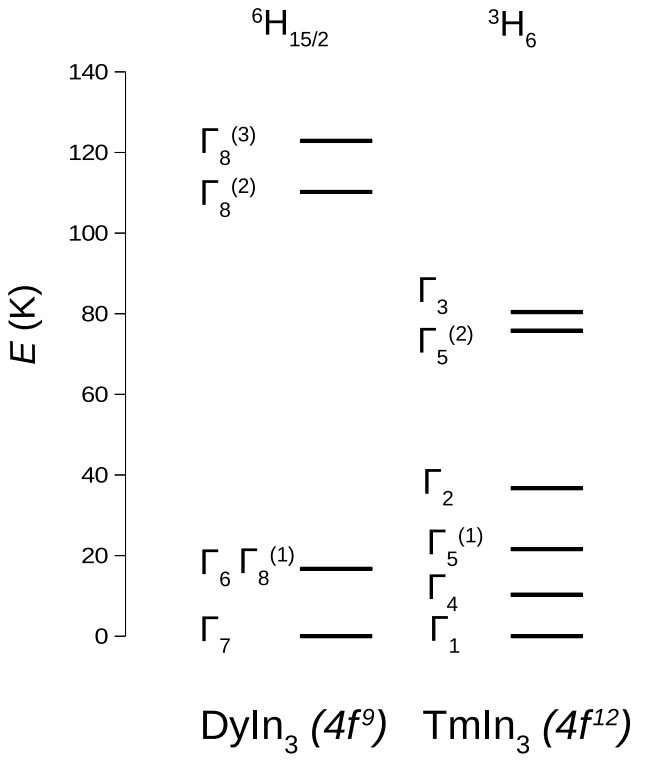

Fig. 1. CF energy levels obtained by simultaneous diagonalization of intraatomic interactions and CF potential (56) using the parameters from Table 1.

like the impurity contribution. At low temperatures it is the ground CF level which determines the s-f contribution $[58,59]$. For $\operatorname{DyIn}_{3}$ the ground state doublet $\Gamma_{7}$ produces a finite contribution at $0 \mathrm{~K}$, while the singlet $\Gamma_{1}$ ground level of $\mathrm{TmIn}_{3}$ does not contribute below the temperature at which the first excited CF level $\Gamma_{4}$ becomes noticeably populated. Thus, in the latter case the whole observed residual resistivity have to originate from the impurity scattering component in the frame of the present model.

In order to test the agreement between the experimental values of the electrical and thermal resistivity and those following from the theoretical formulas of the present paper, we fit the theoretical values to the experimental ones in the paramagnetic phase of the compounds up to $300 \mathrm{~K}$. We consider that there is a good agreement between the experimental and theoretical curves both in the case of $\rho(T)$ and $W(T)$ when one is able to fit both curves with the same and reasonable values of the fitting parameters such as $\rho_{\mathrm{imp}}, \rho_{\mathrm{O}}^{\text {(sf) }}, \rho_{\mathrm{O}}^{(\mathrm{ac})}$ and $\rho_{\mathrm{O}}^{(\mathrm{op})}$ of the formulas (1).

In fact, we apply as many as (unavoidable) eight parameters of the equations formed of (1) with (21)-(23), (38), (39) and (49)-(51) to describe the curves $\rho(T)$ and $T W(T)$ to fit them to the experimental data for $\mathrm{DyIn}_{3}$ and $\mathrm{TmIn}_{3}$. These are the coefficients $\rho_{\mathrm{imp}}, \rho_{\mathrm{o}}^{(\mathrm{sf})}, \rho_{\mathrm{o}}^{(\mathrm{ac})}$ and $\rho_{\mathrm{o}}^{(\mathrm{op})}$ in (1), the Debye and Einstein temperatures and the energies $\epsilon_{\mathrm{F}}, \epsilon_{\mathrm{S}}$. The dimensionless parameter $\xi$ has been estimated from equation (30) assuming $\gamma=(0.25)^{1 / 3}$, $\mu=1$ and lattice constants from Table 1 . The number of these parameters seems rather large, however, the same parameters have to be applied to the two equations, the temperature interval is large and the various parameters occur to be effective at various parts of this interval. Furthermore, there are physical restrictions as to the values of certain parameters. For instance, the magnitude of the Debye temperature should be close to that following from 
other type of measurements or calculations, i.e. the possibly lowest value in the rank of $150-300 \mathrm{~K}$. There are also restrictions on the value of $\epsilon_{\mathrm{F}}$ which should be such in order not to lead to too great number of the current carriers and not too small in order the requirements of the approximation were fulfilled (see the argumentation above Section 3.2.1). Finally, the values of the obtained fitting parameters should be consistent with those obtained for their counterparts of the $\mathrm{REIn}_{3}$ series.

While representing experimental data of [6] as $\rho(T)$ and $T W(T)$ our attention was focused on the difference in curvature between $\rho(T)$ and $T W(T)$ manifesting for both compounds within the whole temperature range. It is worth to add here that by using (38) and (39) we are able to fit more satisfactory than in [4] the curvature of the theoretical $T W(T)$ curve to that of the experimental one. It is due to the fact that in [4] the fitting of both $\rho(T)$ and $T W(T)$ was based on the same function behaving like $\mathcal{G}_{11}\left(t_{\mathrm{E}}\right)$ (see Fig. 2) and presented in [12] and [10]. Figure 2 shows that curvature of $\mathcal{G}_{22}\left(t_{\mathrm{E}}\right)$ becomes more pronounced for "more classical metals", i.e. for higher values of $\epsilon_{\mathrm{F}}$ and the magnitude of the optical phonon energy $T_{\mathrm{E}}$ is also responsible for the effect.

The procedure of the fitting of equation (1) to experimental data was performed in several cycles in order to assure an expected accuracy and consistency. In each cycle $\rho(T)$ was fitted first, then the obtained parameters were used as initial ones to the fitting of $T W(T)$ with the same constraints as applied to the electrical resistivity. The results are presented in Figure 3 and Table 2 show the relevant parameter sets derived for the measured $\rho(T)$ and $T W(T)$. It is seen that the parameters are quite consistent for DyIn ${ }_{3}$. It means that obtained values of the fitting parameters are the same in case of $\rho(T)$ and $T W(T)$ and they fall within the accepted limits. In case of $\operatorname{TmIn}_{3}$ we were not able to assure similar agreement. The values of $T_{\mathrm{D}}$ and $T_{\mathrm{E}}$ are visibly higher for $T W(T)$ than these fitted to $\rho(T)$. In both cases they are higher than the values obtained for $\mathrm{DyIn}_{3}$. Notice, however, that the values of $T_{\mathrm{D}}$ and $T_{\mathrm{E}}$, i.e., of the phonon energies are related to the curvature of the experimental $T W(T)$ dependence in the whole temperature range.

The material constants obtained from the fitting for $\operatorname{TmIn}_{3}$ are shown in the penultimate column of Table 2. Nearly all the parameters are of considerably greater value. This suggests that the model approximations are too simple to describe well this compound and the oversimplification mainly concerns the phonon system. There is also a possibility that experimental data are incorrect but we rather exclude it since the measurements were made twice with two different apparatus.

In search of the material constants for $\mathrm{TmIn}_{3}$ being of the nearly the same magnitude as those for $\mathrm{DyIn}_{3}$ we scaled the experimental results $T W(T)$ for $\mathrm{TmIn}_{3}$ so as to obtain at high temperatures the same magnitude of the Lorenz number of the same value as that of $\mathrm{DyIn}_{3}$. In this way we reduced for all the temperatures the values of $T W(T)$ by the factor 0.338 and obtained the material constants nearly the same as in case of $\mathrm{DyIn}_{3}$. In fact, we expected similar values of the material constants in case of both the compounds and the fact that this is obtained
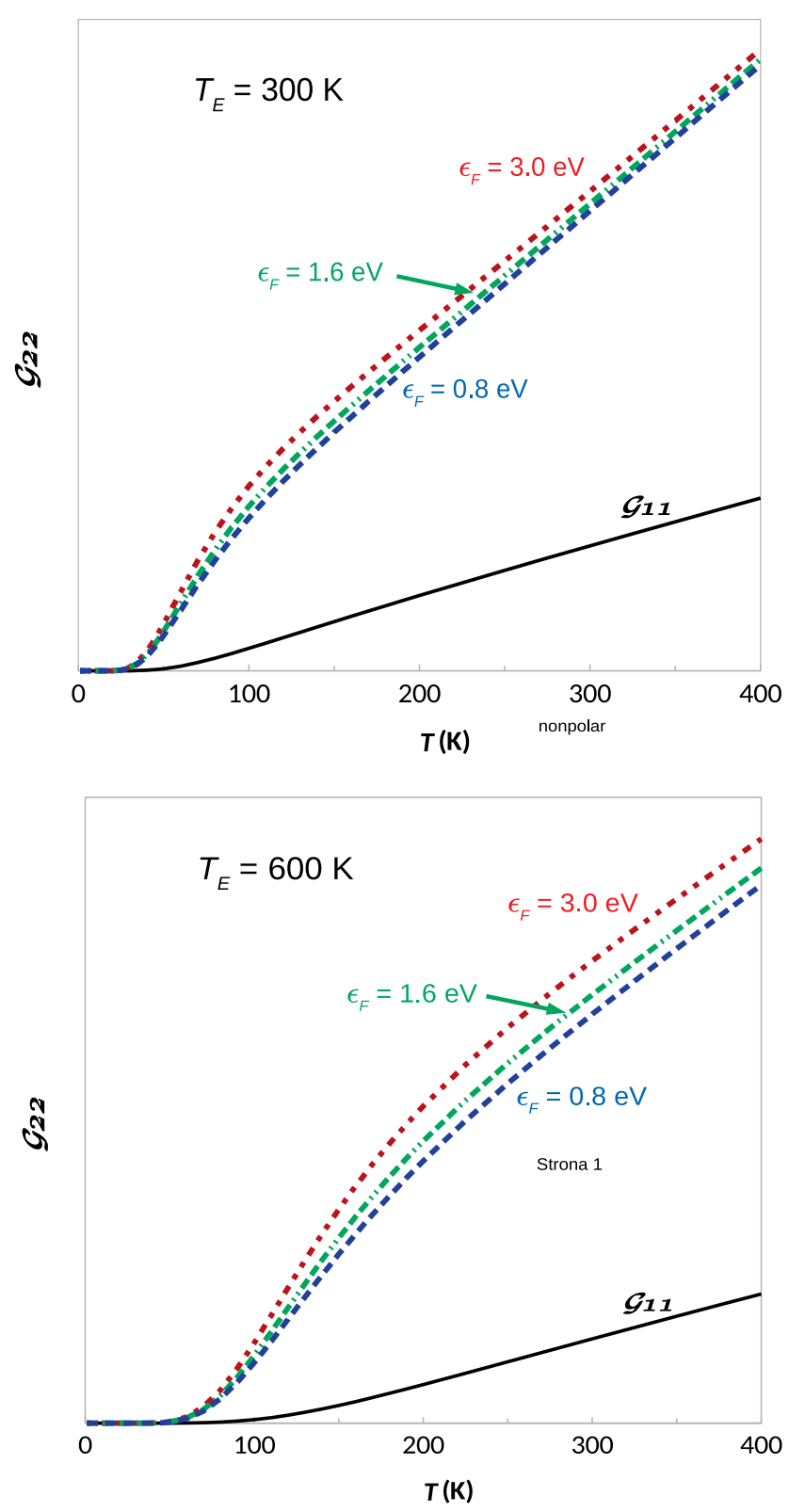

Fig. 2. $\mathcal{G}_{11}\left(t_{\mathrm{E}}\right)$ and $\left.\mathcal{G}_{22}\left(t_{\mathrm{E}}\right)\right],(38)-(39)$, as the function of $T$ for $T_{\mathrm{E}}=300$ and $600 \mathrm{~K}$ and three different Fermi energies. According to the expectation the temperature at which maximum of the curvature is observed relates to $T_{\mathrm{E}}$. Moreover it is more pronounced for both higher $T_{\mathrm{E}}$ and $\epsilon_{\mathrm{F}}$.

after reducing the values of $T W(T)$ for $\mathrm{TmIn}_{3}$ is at present not fully understandable for us. Therefore, another fitting strategies were probed. Satisfactory precision of the fitting to the experimental $T W(T)$, just like that seen in Figure 3 , was obtained by minimizing $\rho_{\mathrm{imp}}$ to get it as close the value of this parameter derived from the electrical resistivity for this compound as possible. The results are shown in the last column of Table 2.

As it is seen, $\rho_{\mathrm{imp}}$ and $\rho_{\mathrm{o}}^{(\mathrm{ac})}$ in this set are quite close to the values obtained from the fitting of the electrical resistivity. The phonon energies $T_{\mathrm{D}}$ and $T_{\mathrm{E}}$ are also closer to their counterparts. Unfortunately, $\widetilde{\rho_{\mathrm{o}}^{(\mathrm{op})}}=\left(8 / \xi^{2}\right) \rho_{\mathrm{o}}^{(\mathrm{op})}$ and 
Table 2. The parameters obtained by the fitting of equation (1) to the electrical and thermal resistivities for DyIn 3 and $\operatorname{TmIn}_{3}$. Two different sets of parameters fitted to the measured $T W(T)$ are presented for $\operatorname{TmIn}_{3}$. Values given in the parentheses were obtained for scaled experimental curve. For further explanation see the main text. To maintain correspondence with the convention used previously after reference [9] we replace here $\rho_{\mathrm{o}}^{(\mathrm{op})}$ with $\widetilde{\rho_{\mathrm{O}}^{(\mathrm{op})}}=\left(8 / \xi^{2}\right) \rho_{\mathrm{O}}^{(\mathrm{op})}$ where $\xi$ is given by equation (30) calculated for $\mu=1$.

\begin{tabular}{|c|c|c|c|c|c|c|}
\hline Paramete & s derived from & $\begin{array}{l}\operatorname{DyIn}_{3} \\
\rho(T)\end{array}$ & $T W(T)$ & $\begin{array}{l}\operatorname{TmIn}_{3} \\
\rho(T)\end{array}$ & $T W(T)$ & \\
\hline$\rho_{\mathrm{imp} / \mathcal{L}_{\mathrm{o}}}$ & {$\left[\mathrm{m} \mathrm{K}^{2} / \mathrm{W}\right]$} & 16 & 16 & 52 & $241(81)$ & 73 \\
\hline$\underline{\rho_{\mathrm{o}}^{(\mathrm{ac})} / \mathcal{L}_{\mathrm{o}}}$ & & 422 & 473 & 407 & $1284(430)$ & 669 \\
\hline$\rho_{\mathrm{o}}^{(\mathrm{op})} / \mathcal{L}_{\mathrm{o}}$ & & 39 & 32 & 38 & $76(27)$ & 130 \\
\hline$\rho_{\mathrm{O}}^{(\mathrm{sf})} / \mathcal{L}_{\mathrm{o}}$ & & 11 & 17 & 19 & $242(83)$ & 417 \\
\hline$T_{\mathrm{D}}$ & {$[\mathrm{K}]$} & 158 & 163 & 197 & $368(362)$ & 299 \\
\hline$T_{\mathrm{E}}$ & & 390 & 474 & 400 & $665(653)$ & 563 \\
\hline$\epsilon_{\mathrm{s}}$ & {$[\mathrm{K}]$} & & 1.2 & & $5.0(3.2)$ & 3.5 \\
\hline$\epsilon_{\mathrm{F}}$ & {$[\mathrm{eV}]$} & & 2.2 & & $2.0(2.6)$ & 2.6 \\
\hline
\end{tabular}

$\rho_{\mathrm{o}}^{(\mathrm{sf})}$ is five and as much as twenty times larger than their electrical resistivity counterparts. The result is related to the considerable less magnitude of the Lorenz number seen in Figure 3 of [6] for $\operatorname{TmIn}_{3}$. Thus, this scenario also requires further theoretical and experimental investigations. First, inclusion of the excited electron configuration $\mathrm{f}^{n} \Rightarrow \mathrm{f}^{n-1} \mathrm{~d}$, admission of some degree of delocalization of the $4 \mathrm{f}$ electrons and reduction of the intra-atomic Coulomb and spin-orbit coupling constants.

It is clear from the presented formulas for particular components of the transport characteristics under consideration that the adjustable parameters are not quite independent. It concerns especially those describing the two contributions of the scattering by phonons. Therefore, the obtained values of the parameters should be handled with caution as a reference to possible further estimations of material constants. Apart from the Debye, Einstein and Fermi energy, which are directly fitted to the experimental data, the obtained magnitudes of other parameters shed some light on the value of other pertinent quantities (see also Appendix C). The constant $C$, equation (55), which can be extracted directly form the experiment, allows to determine the interaction s-f constant, $j_{\text {ex }}$, which should not differ too much along the series. Unfortunately, the value of $C$ obtained here from the parameters listed in Table 2, similarly as the one determined directly from experiment, as seen from Figure 2 in reference [6], turns out to be over ten times larger for $\mathrm{TmIn}_{3}$ than for $\mathrm{DyIn}_{3}$. This leads to over three times larger values of $j_{\text {ex }}$. The ratio of $j_{\text {ex }}$ for $\operatorname{TmIn}_{3}$ and $\mathrm{DyIn}_{3}$ even increases to over five if derived from $T W(T)$. We checked that this behavior is not caused by omitting the non-spherical Coulomb scattering $[58,60-62]$ which leads to much the same temperature dependence of the electrical resistivity as the s-f one [58].

\section{Final remarks}

By using the models and the approximations, which we presented in successive sections of this paper, we obtained simple formulas for additive contributions to the electrical and thermal resistivity. The method can be applied to a description of the transport in certain felectron rare earth intermetallics or simpler metals if the magnetic s-f contribution is neglected. The presented here approach is a development, simplification and correction of several earlier papers of one of the authors. The development concerns taking into account the scattering of the conduction electrons by optical phonons and while considering the s-f scattering also the crystal field. In [53] the crystal field was considered within oversimplified two-level model. In [4] the final formula for many-level system was merely presented. In the present paper we derive it and by adding the terms responsible for the scattering by optical phonons to the result of [4] we manage to get a better quantitative correspondence of the temperature dependence of the thermal resistivity to the experimental results for $\mathrm{DyIn}_{3}$. According our knowledge the influence of the optical phonons on the transport in metals was restricted up to now only to an analysis of temperature dependence of the electrical resistivity of certain metals [9-11].

One can expect that the theory such as in the present paper, it means based on the free electron approximation and simple models, can be hardly applied to quantitative analysis of experimental data of the transport coefficient in metals. Nevertheless, we attempted this task in [4] and in the present paper with quite satisfactory result in the case of $\mathrm{DyIn}_{3}$ and less satisfactory concerning the derived material constants for $\mathrm{TmIn}_{3}$. The reason is not clear for us, since one rather expects that differences in the value of the lattice constant and in the number of the f-electrons between RE-ions of these two examined compounds should not imply large changes in the probabilities of the scattering. In fact, the changes concern the heat carrier scattering rather than the electric charge scattering since this is the thermal conductivity which considerably vary between that of $\mathrm{DyIn}_{3}$ and $\mathrm{TmIn}_{3}$. Since phonons as heat carriers in these metallic systems can be excluded, we are inclined to consider that the reason of the discrepancy in $\operatorname{TmIn}_{3}$ is related to a subtle role of the conduction electrons in the scattering. Namely, if the value of the Fermi energy counted from the bottom of the conduction band is not sufficiently large, the upper limit of the integrals of which the scattering matrices are composed is not simply related to the Debye temperature. 

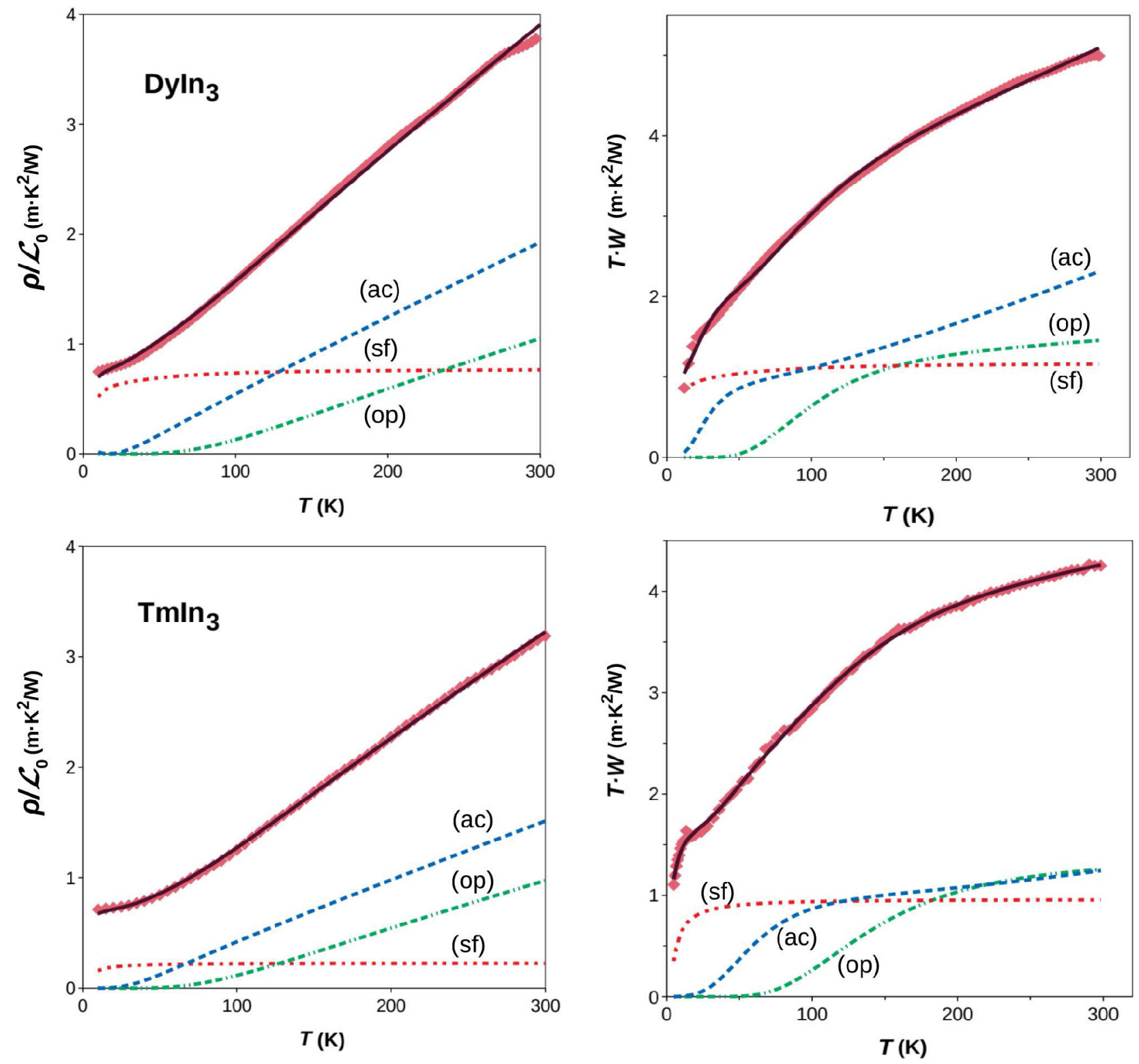

Fig. 3. Experimental (diamonds) versus theoretical (solid line) temperature dependency of the electrical and thermal resistivity for $\mathrm{DyIn}_{3}$ and $\mathrm{TmIn}_{3}$. The influence of the scattering on acoustic phonons (ac), optical phonons (op) and due to the s-f interaction (sf) are shown. The lattice imperfections lead to the temperature-independent contribution which is seen as the gap between the model curve and the s-f one at the low temperatures in which the phonons are ineffective. Note different temperature ranges of non-linearity of the particular scattering mechanisms seen for $T \cdot W(T)$.

Furthermore, the formulas (7) and (8) cease to be valid and one has to apply their more general counterparts, namely equations (7) and (9) of [13] (or see Sect. III of [28]). It means that the electrical and thermal resistivities are dependent on all the components of the scattering matrix $P_{i j}$, microscopic electrical and thermal currents and the temperature dependent chemical potential. The formulas (1) are also no longer valid. Therefore, there is a disagreement between the material parameters describing the electrical and thermal resistivity following from (1) when one fits the values of these transport coefficient to experimental data.

Qualitative studies of the thermal conductivity of $\mathrm{REIn}_{3}$ compounds were also presented a few years ago in [63], where the author used the variation method with the electrical resistivity expressed by $\mathcal{P}_{11}$ and the thermal resistivity by $\mathcal{P}_{22}$. In this work the influence of the scattering by phonons was restricted to acoustic phonons with the application of the Wilson formula (24). The influence of the s-f scattering was taken into account by using a formula following from the consideration in [52], where instead of our expression of the transition probability (44) by the correlation function - the authors used the generalized susceptibility. While using this approach, the author of [63] obtained that $\mathcal{P}_{22}=(k T)^{2} \mathcal{P}_{11}$ (compare our formulas (49)-(51) what seems to be incorrect, probably because of not taking into account all terms of the Sommerfeld expansion (see (48)). Of the three REIn ${ }_{3}$ compounds 
analyzed in [63] only $\mathrm{TmIn}_{3}$ was considered by us in the present paper. However, the different way in which the crystal field eigen energies and eigen functions were introduced into consideration in [63] and the application of the formula $\mathcal{P}_{22}=(k T)^{2} \mathcal{P}_{11}$ cause that the qualitative analysis in this paper for $\mathrm{TmIn}_{3}$ and our present results cannot be compared.

The Wilson formula (24), which is mentioned above, imply the necessity of taking into account U-processes (or Umklapp scattering), which were neglected in its derivation. This is because the function can be decreasing in some temperature interval. Terms added to this function describing the U-processes improve the shortcoming of the Wilson formula. According later investigations [7] the electron-phonon scattering in alkali-like metals is dominated by $\mathrm{N}$-processes provided that the scattering on dislocations enhancing the Umklapp processes can be neglected. If we take the statement of [7] for granted, we can consider that the formula (23) is correct and that the U-processes have not to be taken in account. In any case the function (23) is increasing for arbitrary values of the material constants (see Fig. 3).

Rare earth materials can find applications in certain electronic devices and knowledge of mutual influence of their thermal and electrical resistivity at various temperatures seems necessary. By examining monocrystalline samples we are able to get information on their transport properties which are free of the influence of the crystal grain structure. The primary aim of our present considerations was supplying a simple theoretical approach allowing to express the electrical resistivity, thermal resistivity and also Seebeck coefficient in terms of analytic functions derived for all these coefficients in the same approximation and capable of studying the influence of the material constants on their temperature dependence. Such studies are useful to understanding the transport properties of a series of intermetallics such as $\mathrm{REIn}_{3}$ or $\mathrm{REAl}_{2}$ [64]. We managed to present such formulas and furthermore showed the way in which the crystal field influence on the temperature dependence of these transport coefficients can be examined.

Nowadays, ab initio methods based on a densityfunctional theory (DFT) are used to understanding thermal transport processes in certain materials or even to modeling materials of future applications $[65,66]$. Since no such method has been developed to study f-electron materials and there is still an inaccuracy in DFT-based force calculations up to now the method proposed in the present paper and its improvements (e.g. for not a strongly degenerate electron gas, Eqs. (3.4)-(3.6) of [28]) seems to be useful tool in studying these materials.

The authors are grateful to Prof. M. Ausloos for critical reading of the manuscript. They also acknowledge Dr. J. Ulner for paying attention to certain items of the literature.

\section{Author contribution statement}

K. Durczewski performed transport analytical calculations and prepared the related part of the manuscript.
Z. Gajek determined the electronic structure of the localized electrons, adjusted the phenomenological parameters to the experimental data and wrote the corresponding part of the paper. J. Mucha provided the experimental data. All the authors have read and approved the final manuscript.

Publisher's Note The EPJ Publishers remain neutral with regard to jurisdictional claims in published maps and institutional affiliations.

Open Access This is an open access article distributed under the terms of the Creative Commons Attribution License (http://creativecommons.org/licenses/by/4.0), which permits unrestricted use, distribution, and reproduction in any medium, provided the original work is properly cited.

\section{Appendix A: Remark on results of papers $[13,27,28]$}

In [4] we applied the final formulas for $\mathcal{P}_{i j}(T)$ which were derived in [28] and applied in [13] to illustrate the temperature dependence of the relevant transport coefficients. The mentioned final formulas followed from the form of $\mathfrak{C}(\mathbf{k}, \mathbf{q})$ applied after [27] where other approximation methods were applied than in [28] and results not in the terms of the standard functions were obtained. The results of $[27,28]$ are a consequence of the erroneous assumption that one can take into account in the calculations $\mathfrak{C}(\mathbf{k}, \mathbf{q})=2 \mathfrak{C}_{\mathrm{a}}(\mathbf{k}, \mathbf{q})$ instead of $\mathfrak{C}_{\mathrm{a}}(\mathbf{k}, \mathbf{q})+\mathfrak{C}_{\mathrm{e}}(\mathbf{k}, \mathbf{q})$. This assumption is valid in case of the electrical resistivity, it means while calculating $\mathcal{P}_{11}(T)$. In [27] it was generalized to the remaining matrix elements by falsely applying the principle of the detailed balancing $[29,46]$ (see Eq. (3.4) in [27]).

\section{Appendix B: The metallic limit of certain pertinent integrals}

The evaluation of the integrals with respect the electron energy such as those in (31) consists in taking advantage of the identity (15), (16), the integration by parts and finally in finding the metallic limit $z=\zeta / k_{\mathrm{B}} T \rightarrow \infty$. Consider the functions $\left.\Phi_{\mathrm{a}}(w), \Phi_{\mathrm{e}}(w)\right)$ (36), (40) not to depend on $w=\epsilon / \hbar \omega_{0}$. It means that $\phi_{\mathrm{a}}(w), \phi_{\mathrm{e}}(w)$ in (36) and (40) are not neglected. The task is then reduced to considering the following integrals

$$
\begin{gathered}
\mathcal{N}_{\circ}^{\prime} \int_{0}^{\infty} \mathrm{d} \epsilon f_{0}(\epsilon)\left[1-f_{0}\left(\epsilon_{+}\right)\right](\epsilon-\zeta)^{m} \\
=\mathfrak{F}_{0} \int_{-z}^{\infty} \mathrm{d} y y^{m} \mathfrak{F}(y, p) \\
\mathcal{N}_{\circ}^{\prime \prime} \int_{0}^{\infty} \mathrm{d} \epsilon f_{0}(\epsilon)\left[1-f_{0}\left(\epsilon_{-}\right)\right](\epsilon-\zeta)^{m} \\
=-\mathfrak{F}_{0} \int_{-z+p}^{\infty} \mathrm{d} y y^{m} \mathfrak{F}(y,-p)
\end{gathered}
$$


where $\mathcal{N}_{\circ}^{\prime}$ and $\mathcal{N}_{\circ}^{\prime \prime}$ are the quantities defined below equation (31) and the functions in the right-hand side integrals are defined in (15), (16) and $\epsilon_{+}=1+\hbar \omega_{0}, \epsilon_{-}=1-\hbar \omega_{0}$ and $\left.\mathfrak{F}_{0}=1 /\left[\mathrm{e}^{p}-1\right)\left(1-\mathrm{e}^{-p}\right)\right]$. For $m=0,1,2$ the limit values of the integrals of the right-hand side of (B.1) are (see, e.g. Appendix B of [28])

$$
\begin{gathered}
\int_{-\infty}^{\infty} \mathrm{d} y \mathfrak{F}(y, u)=u, \quad \int_{-\infty}^{\infty} \mathrm{d} y y \mathfrak{F}(y, u)=-\frac{1}{2} u^{2}, \\
\int_{-\infty}^{\infty} \mathrm{d} y y^{2} \mathfrak{F}(y, u)=\frac{1}{3} u^{3}+\frac{\pi^{2}}{3} u .
\end{gathered}
$$

The above approximation is sufficient to to calculate the contribution of the functions $\Phi_{\mathrm{a}}(w), \Phi_{\mathrm{e}}(w)$ to $\mathcal{P}_{i j}(T)$. The matrix element which is essential for the magnitude of the thermoelectric power is proportional to

$$
\int_{-\infty}^{\infty} \mathrm{d}(y+p) \mathfrak{F}(y, u)-\int_{-\infty}^{\infty} \mathrm{d}(y-p) \mathfrak{F}(y, u)=0,
$$

and thus $\mathcal{P}_{12}(T)=0$.

The functions $\Psi_{\mathrm{a}}(w), \Psi_{\mathrm{e}}(w)$ contribute to the equation (31) without the factors $(\epsilon-\zeta)^{2}$ and $\epsilon-\zeta$. It enables simply and generally to calculate their final contribution to the functions $\mathcal{G}_{22}\left(t_{\mathrm{E}}\right)$ which is

$$
\mathcal{G}_{22}^{(\Psi)}(p)=\frac{p^{2}}{\left(\mathrm{e}^{p}-1\right)\left(1-\mathrm{e}^{-p}\right)}\left[\widetilde{\Psi_{\mathrm{e}}}(p)-\widetilde{\Psi_{\mathrm{a}}}(-p)\right]
$$

where $\widetilde{\Psi_{\mathrm{a}}}(u)=\int_{0}^{u} \mathrm{~d} y \Psi_{\mathrm{a}}(y), \widetilde{\Psi_{\mathrm{e}}}(u)=\int_{0}^{u} \mathrm{~d} y \Psi_{\mathrm{e}}(y)$.

\section{Appendix C: Material constants $\rho_{\mathrm{o}}^{(\mathrm{ac})}, \rho_{\mathrm{o}}^{(\mathrm{op})} \rho_{\mathrm{o}}^{(\mathrm{sf})}$ of equations (1)}

In Section 6 we fit equation (1) to the experimental data by using the coefficients of the dimensionless functions of $\rho(T), W(T)$ and certain parameters of these functions. Therefore, in fact the knowledge of the explicit forms of these coefficients such as follow from the presented theoretical considerations based on the Ziman variational method is not needed. However, these forms show how the values of the fitted coefficients are interrelated. These interrelations cause that under the influence of a variation in the magnitude of a single fitting parameter there can occur a considerable change in the value of the remaining ones. On the other hand, some the explicit form of these coefficients indicates relations between the values of these coefficients for different members of the $\mathrm{REIn}_{3}$ series.

The magnitude of the material constant in front dimensionless linear combinations of the Bloch-Grüneisen functions, $\mathcal{F}_{i j}\left(t_{\mathrm{D}}\right)$ such as in (20) was derived in the Appendix B of [13]. For completeness we also present here. It reads

$$
\mathcal{P}_{0}^{(\mathrm{ac})}=\frac{1}{4 \pi^{3}} \frac{V_{\mathrm{c}} E_{1}^{2}}{12 M v_{\mathrm{s}}}\left(\frac{2 m}{\hbar^{2}}\right)^{2}\left(\frac{\hbar}{m}\right)^{2} q_{\max }^{5},
$$

where $\hbar v_{\mathrm{s}} q_{\max }=k_{\mathrm{B}} T_{\mathrm{D}}$ and $q_{\max }=2 q_{\mathrm{D}}$ with the radius of the Debye sphere $q_{\mathrm{D}}$. The expression for the value of the electrical resistivity in (1) is obtained from the relation

$$
\rho_{0}^{\text {(ac) }}=\frac{\mathcal{P}_{\mathrm{o}}^{\text {(ac) }}}{e^{2} U_{0}^{2} \epsilon_{\mathrm{F}}^{3}}, \quad U_{0}^{2}=\left(\frac{1}{6 \pi^{2}}\right)^{2}\left(\frac{2 m}{\hbar^{2}}\right)^{5}\left(\frac{\hbar}{m}\right)^{4}
$$

what yields

$$
\rho_{0}{ }^{(\mathrm{ac})}=\frac{3 \pi}{4} \mu^{2}\left(\frac{m_{\mathrm{o}}}{\hbar^{2} e^{2}}\right) \frac{m_{\mathrm{o}}}{M} V_{\mathrm{c}} E_{1}^{2} \widetilde{\rho}_{\mathrm{o}}^{(\mathrm{ac})}
$$

where $m_{\mathrm{o}}$ denotes the mass of the free electron $\mu=m / m_{\mathrm{o}}$, $m_{\mathrm{o}} / \hbar^{2} e^{2} \approx 9.113 \cdot 10^{3} \mathrm{eV}^{-2} \mathrm{~nm}^{-3}$ and $V_{\mathrm{c}}$ stands for the volume of the primitive cell. The latter factor in (C.3) is

$$
\widetilde{\rho}_{0}^{\text {(ac) }}=\left(\frac{q_{\max }}{2 k_{\mathrm{F}}}\right)^{6}\left(v_{\mathrm{s}} q_{\max }\right)^{-1}=\left(\frac{q_{\max }}{2 k_{\mathrm{F}}}\right)^{6} \hbar\left(k_{\mathrm{B}} T_{\mathrm{D}}\right)^{-1}
$$

where $2 k_{\mathrm{F}}$ is the caliper of the Fermi surface and $q_{\max }$ the caliper of the Debye sphere. The factors $\left(v_{\mathrm{s}} q_{\mathrm{D}}\right)^{-1}$ and $\hbar\left(k_{\mathrm{B}} T_{\mathrm{D}}\right)^{-1}$ are of the dimensionality $\Omega \mathrm{cm}$. For $T_{\mathrm{D}}=200 \mathrm{~K}$ the magnitude of $\hbar\left(k_{\mathrm{B}} T_{\mathrm{D}}\right)^{-1}$ is about $3.347 \cdot 10^{-2} \Omega \mathrm{cm}$.

Similar material constants correspond to nonpolar optical phonons. Obviously, in this case one has to replace $\mathcal{P}_{0}^{(\text {ac) }}$ with $\mathcal{P}_{0}^{(\mathrm{op})}$ and $\widetilde{\rho}_{0}^{\text {(ac) }}$ with $\widetilde{\rho}_{0}^{(\mathrm{op})}$. In the formulas for the latter quantities one should change $q_{\max }^{6}$ for $q_{\max }^{4} G_{0}^{2}$ and $\left(k_{\mathrm{B}} T_{\mathrm{D}}\right)^{-1}$ for $\left(k_{\mathrm{B}} T_{\mathrm{E}}\right)^{-1}$. It is worth reminding here that the quantity $2 q_{\mathrm{D}}=k_{\mathrm{B}} T_{\mathrm{D}} /\left(\hbar v_{\mathrm{s}}\right)$ used in case of the acoustic phonons should also be replaced with one following from the magnitude of the lattice constant $a$ of the cubic lattices. In the case of the simple cubic lattice, we have $q_{\mathrm{D}}=\left(6 \pi^{2}\right)^{1 / 3} /(\gamma a)$ with $\gamma=1$. For the the bcc lattice, the value of $\gamma$ is $(0.5)^{1 / 3}$ and $\gamma=(0.25)^{1 / 3}$ in case of the fcc lattice.

In case of the scattering by polar optical phonons we have

$$
\mathcal{P}_{\mathrm{o}}^{\text {(op) }}=\frac{1}{6}\left(\frac{2 m}{\hbar^{2}}\right)^{3}\left(\frac{\hbar}{m}\right)^{2} \frac{e^{4} \hbar}{d^{3} M^{\prime}},
$$

where $M^{\prime}$ is the mean harmonic mass $1 / M^{\prime}=1 / M^{+}+$ $1 / M^{-}$of the masses $M^{+}$and $M^{-}$of two vibrating ions of the opposite sign which keep the distance $d$ apart [18]. By using similar relations as (C.2) we obtain

$$
\rho_{0}^{(\mathrm{op})}=12 \pi^{4}\left(\frac{e^{2} m_{\mathrm{o}}^{2}}{\hbar^{3}}\right)\left(\frac{m_{\mathrm{o}}}{M^{\prime}}\right)\left(\frac{\mu^{3}}{d^{3} k_{\mathrm{F}}^{6}}\right),
$$

where $e^{2} m_{\mathrm{o}}^{2} / \hbar^{3} \approx 14.69 \Omega \mathrm{cm} / \mathrm{nm}^{3}$.

For the s-f interactions the magnitudes of the material constants such as these above are

$$
\begin{array}{r}
\mathcal{P}_{\mathrm{o}}^{(\mathrm{sf})}=\frac{4}{3} \frac{j_{\mathrm{ex}}^{2}(g-1)^{2} V}{\hbar N_{4 \mathrm{f}}}\left(\frac{m}{\hbar^{2}}\right)^{2}\left(\frac{\hbar}{m}\right)^{2} k_{\mathrm{F}}^{4} \\
\rho_{0}^{\text {(sf) }}=6 \pi^{4} \mu(g-1)^{2}\left(\frac{j_{\mathrm{ex}}^{2}}{\epsilon_{\mathrm{F}}}\right) V_{\mathrm{f}}\left(\frac{m_{\mathrm{o}}}{e^{2} \hbar}\right),
\end{array}
$$

where $V_{\mathrm{f}}=V / N_{4 \mathrm{f}}$ is the volume per a rare earth ion and $m_{\mathrm{o}} / e^{2} \hbar \approx 0.5399 \Omega \mathrm{cm} \mathrm{nm}^{-3} \mathrm{eV}^{-3}$. 


\section{References}

1. T. Kasuya, in Magnetism, vol. IIB, edited by G.T. Rado, H. Suhl (New York Academic Press, New York, 1966), pp. 215-294

2. J. Pierre, in Magnetism of Metals and Alloys, edited by M. Cyrot (North-Holland Publishing Company, Amsterdam, 1980), p. 245

3. J. Mulak, Z. Gajek, The Effective Crystal Field Potential (Elsevier, 2000)

4. K. Durczewski, Z. Gajek, J. Mucha, Phys. Status Solidi B 251, 2265 (2014)

5. Z. Tong, S. Li, X. Ruan, H. Bao, Phys. Rev. B 100, 144306 (2019)

6. J. Mucha, J. Phys. C.: Condens. Matter 18, 1427 (2006)

7. C. Uher, in Thermal Conductivity, Theory, Properties and Applications, edited by T.M. Tritt (Kluwer Academic/Plenum Press, New York, 2004), pp. 21-91

8. G.D. Mahan, L. Lindsay, D.A. Broido, J. Appl. Phys. 116, $245102(2014)$

9. C. King, H. Kirsch, T. Geballe, Solid State Commun. 9, 907 (1971)

10. T. Tanaka, T. Akahane, E. Bannai, S. Kawai, N. Tsuda, Y. Ishizawa, J. Phys. C: Solid State Phys. 9, 1235 (1976)

11. P.B. Allen, W.W. Schulz, Phys. Rev. B 47, 14434 (1993)

12. J. Howarth, E.H. Sondheimer, Proc. R. Soc. Lond. A 219, $53(1953)$

13. M. Ausloos, K. Durczewski, J. Ulner, Int. J. Mod. Phys. 15, $237(2001)$

14. Y. Onuki, R. Settai, Low Temp. Phys. 38, 89 (2012)

15. Z. Kletowski, P.J. Markowski, Solid State Commun. 62, 299 (1987)

16. B. Bauer, E. Gratz, G. Adam, J. Phys. F 16, 493 (1986)

17. L.A. Smirnov, V.S. Oskotski, in Handbook on the Physics and Chemistry of Rare Earths, edited by K.A. Gschneider, L. Eyring (Elsevier Publishers B.V., Amsterdam, 1993), pp. 109-224

18. H. Fröhlich, Proc. R. Soc. Lond. A 160, 230 (1937)

19. E.S. Lage, Z. Naturforsch. 37a, 546 (1982)

20. L. van Hove, Phys. Rev. 95, 249 (1954)

21. G. Grimvall, The electron-phonon interaction in metals, selected Topics in Solid State Physics (North-Holland, Amsterdam, 1981)

22. G. Grimvall, Thermophysical properties of materials, selected Topics in Solid State Physics (North-Holland, Amsterdam, 1986)

23. J.M. Ziman, Electrons and Phonons, selected Topics in Solid State Physics (Clarendon Press, Oxford, 1962)

24. M. Kohler, Z. Phys. 125, 679 (1947)

25. M. Kohler, Z. Phys. 126, 481 (1949)

26. A.H. Wilson, The Theory of Metals, 2nd edn. (Cambridge University Press, Cambridge, 1965)

27. K. Durczewski, M. Ausloos, J. Magn. Magn. Mater. 51, 230 (1985)

28. K. Durczewski, M. Ausloos, Phys. Rev. B 53, 1762 (1996)

29. F. Blatt, Physics of Electronic Conduction in Solids (McGraw Hill, New York, 1968)

30. P. Argyres, J. Phys. Chem. Solids 19, 66 (1961)

31. C. Kittel, An Indroduction to Solid State Physics (John Willey, New York, 1996), Appendix J
32. A. Wilson, Proc. Camb. Philos. Soc. 33, 371 (1937)

33. R.E.B. Makinson, Proc. Camb. Philos. Soc. 34, 474 (1938)

34. R. Berman, Thermal Conductivity in Solids (Oxford University Press, Oxford, 1976)

35. A.E. Szukiel, J. Magn. Magn. Mater. 405, 324 (2016)

36. H. Leung, F. Kus, N. McKay, J.P. Carbotte, Phys. Rev. B 16, 4358 (11977)

37. R.S. Sorbello, J. Phys. F 4, 503 (1974)

38. R.S. Sorbello, Phys. Kondens. Materie 19, 303 (1975)

39. P.B. Allen, Phys. Rev. B 17, 3725 (1978)

40. F.J. Pinski, P.B. Allen, Phys. Rev. B 23, 5080 (1981)

41. Z. Tong, H. Bao, Int. J. Heat Mass Transfer 117, 972 (2018)

42. L. Lindsay, A. Katre, A. Cepellotti, N. Mingo, J. Appl. Phys. 126, 050902 (2019)

43. L.A. Falkovsky, Phys. Rev. B 66, 020302(R) (2002)

44. L.A. Falkovsky, J. Exp. Theor. Phys. 95, 354 (2002)

45. P. Vogl, in Physics of Nonlinear Transport in Semiconductors, edited by D.K. Ferry, J.R. Barber, C.J. Jacobini (Plenum Press, New York, 1980), pp. 75-115

46. P.N. Butcher, in Electrons in Crystalline Solids, edited by G. Chiarotti (International Atomic Agency, Vienna, 1973), pp. 103-166

47. C. Kittel, Quantum Theory of Solids (John Willey, New York - London, 1963), Chap. 7

48. T. Kasuya, Progr. Theor. Phys. 16, 58 (1956)

49. T. Kasuya, Progr. Theor. Phys. 22, 227 (1959)

50. S.H. Liu, Phys. Rev. 132, 589 (1963)

51. N.H. Andersen, P.E. Gregers-Hansen, E. Holm, H. Smith, O. Vogt, Phys. Rev. Lett. 32, 1321 (1974)

52. N.H. Andersen, J. Jensen, O. Splittorff, O. Vogt, Phys. Rev. B 21, 189 (1980)

53. A. Rassili, K. Durczewski, M. Ausloos, Phys. Rev. B 58, 5655 (1998)

54. W.T. Carnall, G.L.G.K. Rajnak, R.S. Rana, J. Chem. Phys. 90, 3443 (1989)

55. B.G. Wybourne, Spectroscopic properties of rare earths (Interscience, New York, 1965)

56. R.M. Galéra, E. Sole, M. Amara, P. Morin, P. Burlet, A.P. Murani, J. Phys.: Condens. Matter 15, 6269 (2003)

57. A. Deutz, H. Brom, W. Huiskamp, L. de Jongh, K. Buschow, J. Magn. Magn. Mater. 78, 31 (1989)

58. T. Hiraoka, K. Kamihira, J. Phys. Soc. Jpn. 56, 1837 (1987)

59. N.A. Frederick, M.B. Maple, J. Phys.: Condens. Matter 15, 4789 (2003)

60. N. Hessel Andersen, O. Vogt, J. Phys. Colloques 40, C5 (1979)

61. J.A. Blanco, M. Reiffers, D. Gignoux, D. Schmitt, A.G.M. Jansen, Phys. Rev. B 44, 9325 (1991)

62. R. Troć, Z. Gajek, M. Pasturel, R. Wawryk, M. SamselCzekała, Intermetallics 107, 60 (2019)

63. A.E. Szukiel, arXiv:1505.01521v2 [cond-mat.str-el] (2018)

64. E.E. Gratz, H. Nowotny, J. Magn. Magn. Mater. 70, 118 (1987)

65. T. Ma, P. Chakraborty, X. Guo, L. Cao, Y. Wang, Int. J. Thermophys. 41, 9 (2020)

66. S. Li, Z. Tong, H. Baoa, J. Appl. Phys. 126, 025111 (2019) 